\title{
S100A10 has a Critical Regulatory Function in Mammary Tumor Growth and Metastasis: Insights using MMTV-PyMT Oncomice and Clinical Patient Sample Analysis.
}

\author{
Alamelu G Bharadwaj \\ Dalhousie University Faculty of Medicine \\ Margaret L Dahn \\ Dalhousie University Faculty of Medicine \\ Ron-Zong Liu \\ University of Alberta Department of Biological Sciences \\ Patricia Colp \\ Dalhousie University Faculty of Medicine \\ Lynn N Thomas
}

Dalhousie University Faculty of Medicine

Ryan W Holloway

Dalhousie University Faculty of Medicine

Paola A Marignani

Dalhousie University Faculty of Medicine

Catherine KL Too

Dalhousie University Faculty of Medicine

Penelope J Barnes

Dalhousie University Faculty of Medicine

Rosaline Godbout

Cross Cancer Institute

\section{Paola Marcato}

Dalhousie University Faculty of Medicine

David Waisman ( $\nabla$ david.waisman@dal.ca)

Dalhousie University https://orcid.org/0000-0002-5097-9662

\section{Research article}

Keywords: Breast cancer, S100A10 (p11), tumor growth, tumor progression, macrophages, metastasis, carcinoma, mammary gland, triple negative 
Posted Date: August 13th, 2020

DOl: https://doi.org/10.21203/rs.3.rs-57686/v1

License: (c) (i) This work is licensed under a Creative Commons Attribution 4.0 International License. Read Full License

Version of Record: A version of this preprint was published at Cancers on December 7th, 2020. See the published version at https://doi.org/10.3390/cancers12123673. 
1 Full Title: S100A10 has a critical regulatory function in mammary tumor growth and metastasis:

2 Insights using MMTV-PyMT oncomice and clinical patient sample analysis.

3 Short Title: S100A10 (p11) and breast cancer progression

4 Author list: Alamelu G. Bharadwaj ${ }^{1}$, Margaret L. Dahn ${ }^{1}$, Ronzong Liu ${ }^{3}$, Patricia Colp ${ }^{1}$, Lynn N. 5 Thomas $^{2}$, Ryan W. Holloway ${ }^{2}$, Paola A. Marignani ${ }^{2}$, Catherine KL. Too ${ }^{2}$, Penelope J. Barnes ${ }^{1}$, 6 Rosaline Godbout ${ }^{4,}$ Paola Marcato ${ }^{1,3}$ and David M. Waisman ${ }^{1,2} *$

$7 \quad{ }^{1}$ Department of Pathology, Dalhousie University, Halifax, Nova Scotia, Canada

$8{ }^{2}$ Department of Biochemistry \& Molecular Biology, Dalhousie University, Halifax, Nova Scotia, 9 Canada

$10{ }^{3}$ Department of Microbiology and Immunology, Dalhousie University, Halifax, Nova Scotia, 11 Canada

$12{ }^{4}$ Department of Oncology, University of Alberta, Edmonton, Alberta, Canada

Keywords: Breast cancer, S100A10 (p11), tumor growth, tumor progression, macrophages, metastasis, carcinoma, mammary gland, triple negative

\section{Abstract}

Background: Breast cancer is one of the leading causes of cancer deaths in women worldwide. Significant advances have been made in the diagnosis and treatment of breast cancer, treatment of triple-negative and metastatic breast cancer poses significant challenge. Metastasis is a multi-step cascade that involves activation of proteases such as plasmin to facilitate the invasive escape of tumor cells to distant organs. The rate-limiting step in plasmin generation requires the interaction of plasminogen with cell surface plasminogen binding sites. Our laboratory first demonstrated that the plasminogen receptor, S100A10 (p11) was upregulated in many cancer cells and was responsible for much of their plasmin generation. Recently, it was reported that p11 is one of a few genes that are activated when human breast cancer cells metastasize from the primary tumor into the blood and is upregulated during the conversion of breast cancer cells to invasive phenotype. In the current study we have investigated the role of p11 in breast cancer tumor progression.

Methods: We have used MMTV-PyMT a mouse transgenic mammary tumor model to investigate the effects of loss of p11 on spontaneous tumor initiation, growth and progression to invasive carcinoma and metastasis. We used experimental metastasis assays to ascertain the role of stromal p11 in tumor cell extravasation and lung colonization. Genes and cytokines regulated by p11 in the PyMT tumors were assessed by microarray analysis and RT-qPCR. Finally, we employed gene profiling analysis and immunohistochemical staining of breast cancer patient tumors to correlate p11 expression to human breast cancer progression. 
Results: Genetic deletion of p11 resulted in significantly decreased tumor onset, growth rate, and spontaneous pulmonary metastatic burden in the PyMT/p11-KO mice. This phenotype was accompanied by substantial reduction in Ki67 positivity, macrophage infiltration, decreased vascular density in the primary tumors and decrease in invasive carcinoma and pulmonary metastasis. Surprisingly, immunohistochemical analysis of wild-type MMTV-PyMT mice failed to detect p1 1 expression in the tumors or metastatic tumor cells and loss of p11 did not decrease plasmin generation in the PyMT tumors and cells. Furthermore, tumor cells expressing p11 displayed dramatically reduced lung metastasis when injected into p11-depleted mice, further strengthening the stromal role of p11 in tumor growth and metastasis. Transcriptome analysis of the PyMT tumors from p11-KO mice showed marked reduction in genes involved in breast cancer development, progression, and inflammation such as AREG, MUC1 and S100A8. The PyMT/p11KO tumors displayed a remarkable increase in inflammatory cytokines such as IL-6, IL-10 and IFN- $\gamma$. Gene expression profiling from 176 primary breast cancer samples obtained through the CBCF tumor bank showed that p11 mRNA levels were significantly higher in tumors compared to normal tissues. P11 mRNA expression was significantly associated with poor patient prognosis (hazard ratio - 3.34) and significantly elevated in high grade, triple negative (TN) tumors, and tumors with high proliferative index. Evaluation of p11 protein expression in a NSHA cohort of patients revealed substantial upregulation of p11 in cancer tissues compared to normal controls.

54 Conclusions: This is the first study demonstrating the crucial role of p11 in breast tumor development and metastasis. The results emphasize the potential of p11 as a diagnostic and prognostic biomarker in breast cancer.

57

58

59

60

61

62

63

64

65

66

67

68

69

70

71

72

73

74

75

\section{Introduction}

Breast cancer is the leading cause of cancer death among women worldwide. The vast number of cancer-associated deaths are due to metastases rather than primary disease alone. Currently, strategies to treat and eliminate metastasis are limited and challenged by resistance to therapies. Thus, discovering the underlying molecular mechanisms that promote breast cancer metastasis and therapy resistance is critical for identifying novel treatment strategies.

Extracellular proteases promote degradation of the extracellular matrix and form a key component of the cascade of events that contribute to cancer cell invasion and metastasis. (1). (2,3). Increasing evidence have shown elevated expression of proteases plasmin and matrix metalloproteases (MMPs) in breast cancer progression and have been extensively studied with mouse tumor models (4). Plasminogen (Plg), synthesized by the liver is activated to plasmin (Pm) by tissue plasminogen activator (tPA) and urokinase plasminogen (uPA) activator, (reviewed in $(5,6)$ ). This process, normally slow, is accelerated by plasminogen receptors $(\mathrm{PgR})$ on the cell surface. Our laboratory identified S100A10 (p11) as a plasminogen receptor that forms a complex with both plasminogen and plasminogen activator (7-10).

P11 is a multifunctional protein and a member of the S100 proteins. Several extracellular and intracellular functions have been identified for $\mathrm{p} 11$; the plasminogen receptor function is the most 
well studied so far (5). P11, present on the extracellular surface, is complexed with its binding partner annexin A2 (p36). We have reported that p11 regulates plasminogen activation on the cell surface of many cancer cells including fibrosarcoma, colorectal, lung and pancreatic cells $(8,11-$ 16). We have also reported that $\mathrm{p} 11$ regulates the plasmin production of stromal cells including macrophages and endothelial cells (17-19) and that p11-dependent plasmin generation is necessary for macrophage infiltration to the site of inflammation in subcutaneous tumor growth.

Several studies have shown correlations between p11 gene expression and poor prognosis and overall survival in lung (20-22), colorectal, ovarian, kidney, gastric cancers, anaplastic thyroid carcinoma, melanoma and acute lymphoblastic leukemia (reviewed in (12)), and pancreatic ductal adenocarcinoma (PDAC)(16). P11 is upregulated in basal-type breast cancer (23) and during the process of intravasation and epithelial mesenchymal transition (24).

Although we have shown that p11 is important for ectopic tumor growth in both NOD/SCID and syngeneic mouse models for various cancer cell lines such as colorectal, fibrosarcoma, and pancreatic cancer, these studies have limitations. Firstly, they do not reproduce the complex multistep landscape of human oncogenesis. Secondly, the growth, invasion and metastasis of cancer also depends on its interaction with the tumor microenvironment and it is also difficult to distinguish the individual contribution of tumor and stromal cells to cancer progression. To circumvent these experimental challenges, and to advance our understanding of the role of p11 in oncogenesis we have established the MMTV-PyMT (Polyoma Middle T) transgenic breast cancer model in wild-type and p11 knockout mice and have used this double transgenic model to investigate the role of p11 in breast cancer malignancy.

In the MMTV-PyMT transgenic mouse model, mammary gland specific expression of the oncogene PyMT under the MMTV promoter, results in widespread tumor growth in all ten mammary glands and spontaneous metastasis to the lymph nodes and lungs. This occurs with a mean latency of 92 days with high penetrance and almost a 100\% incidence of metastasis. This mouse model is very similar to human breast cancer in that the tumors display histological and molecular characteristics mirroring the progression of human breast cancer (25-27)and have a reactive stroma. This model has been widely used to establish the role of proteolytic activity in cancer cell malignancy. Proteases that affect metastasis in this model system include plasminogen (28,29), uPA (30), MMP-9 and MMP-3 (4,31,32), cathepsin B(33,34) and ADAMTS1(35). Another important feature of the MMTV-PyMT model is that the increased metastatic potential is largely dependent on the presence of macrophages in the primary tumor (36,37). Thus, the MMTVPyMT model is a pertinent model to investigate the functional role of $\mathrm{p} 11$.

In the current study, we conducted a systematic, functional, and correlative analysis of the role of p11 in breast cancer oncogenesis using the MMTV-PyMT transgenic mouse model. We have also performed gene expression profiling and protein expression analysis of human breast cancer tissues. The studies described herein indicate that p11 plays a complex and multifunctional role in breast tumor growth, progression, and metastasis.

\section{Materials and Methods}

115 Mice 
All animal experiments were performed according to protocol approved by University Committee on Laboratory Animals, Dalhousie University, Canada. The C57B16 MMTV-PyMT mice were obtained from Dr. Mak (OCI, Canada). The p11 WT and p11 KO mice were obtained from P. Svenningsson (Karolinska Institutet, Sweden). We employed a sequential breeding strategy to generate PyMT/p11-KO homozygous mice. We crossed the male PyMT/p11-WT mice with the female p11 KO mice to obtain heterozygotes for $\mathrm{p} 11$. The resultant F1 (PyMT/ p11+/-) males were bred with the female (p11+/-) mice. This crossbreeding produced F2 females PyMT/p11-WT, (PyMT/p11+/- and PyMT/p11-KO. Virgin female C57/BL6 mice heterozygous for the MMTVPyMT transgene and homozygous KO (PyMT/p11-KO) or homozygous wild-type (PyMT/p11WT) for the p11 gene were used in our studies. Genotyping of mice was performed by PCR of genomic DNA derived from ear clippings using published primers for PyMT and p11 (Supplement table 1).

\section{Cell culture and reagents}

Py8119 cells were obtained from ATCC and maintained in F12 Kaign's medium (Hyclone, Logan, UT, USA) with 5\% Fetal Clone II serum (Thermofisher Scientific), $1 \%$ penicillin and streptomycin. Py8119 cells were tested for pathogen (Charles River Laboratory) before injecting into the mice. All cells were tested for mycoplasma (Lonza Mycoalert kit) and only mycoplasmafree cells were used.

\section{Whole mount analysis}

The harvested tissues were stretched and placed onto a cassette matrix and fixed overnight in Carnoy's fixative. The tissues were sequentially hydrated with 70, 50, 25\% ethanol followed by a final water wash. They were stained with carmine alum solution for four hours, followed by sequential dehydration in 70, 95 and $100 \%$ ethanol and defatting of the tissues was carried out in two xylene washes for 30 minutes and overnight. The dehydrated and defatted tissues were then mounted on slides using Cytoseal (Richard-Allan Scientific, Thermo Scientific, Waltham, MA, USA) mounting medium. The slides were digitally imaged using a stereomicroscope under similar lighting and contrast conditions (Zeiss microscope).

\section{Tumor measurements}

For spontaneous PyMT tumor model, all the mammary glands were palpated once a week for 2025 weeks to monitor tumor latency and progression. For evaluating the kinetics of tumor growth, the tumors were measured using a Vernier calipers, and tumor volume was determined using the standard calculation for a hemi-ellipsoid; $0.5 \mathrm{ab}^{2}$ where $\mathrm{a}$ is the smaller and $\mathrm{b}$ is the larger diameter. Tumor burden was calculated at the endpoint (20 weeks) by determining the total tumor weight/body weight. We determined the percentage of tumor-free mice using Kaplan Meier analysis (Graph pad prism) in the two PyMT mice groups. Animals were considered tumor-free until a palpable mass $(>4.0 \mathrm{~mm})$ persisted for longer than 4 days. All tumor growth and kinetics data were obtained from three independent experiments. Only virgin female mice were used for all the experiments.

\section{Spontaneous and experimental metastasis quantification}


For evaluation and assessment of lung metastasis, lungs were harvested at 20 weeks, fixed in $10 \%$ buffered formalin for 48 hours and embedded in paraffin. The lungs were cut at $5 \mu \mathrm{m}$ thickness and 3-6 sections from each lung at $100 \mu \mathrm{m}$ apart were stained with H\&E. The stained slides were scanned using Aperio microscope (Leica Biosystems, Concord, Ontario) and number of metastatic foci were counted using Imagescope/Image J. The total metastatic burden was determined using Imagescope/Image $\mathrm{J}$ by tracing the metastatic area as a ratio of the total lung area. For experimental metastasis assay, Py8119 cells were injected via the tail vein at a density of $2.5 \times 10^{5}$ cells. The lungs were harvested 14 days after injection and analyzed for metastatic burden and foci calculation as described for spontaneous metastasis quantification.

\section{Tissue Processing and Immunohistochemistry}

Tumors, mammary glands, and lungs were harvested and fixed in neutral $10 \%$ buffered formalin for 48 hours and stored in $70 \%$ ethanol before embedding in paraffin. The embedded tissues were cut into $5 \mu \mathrm{m}$ thick sections, stained with H\&E for pathological analysis, and adjacent sections were used for immunohistochemistry. The tissue sections were deparaffinized with sequential washes in xylene, $100 \%, 95 \%$ and $70 \%$ ethanol followed by water wash. The details of the antibody dilution, method of chromogen and stain development are provided in the Supplement table 2 .

Antigen retrieval was performed by heat treatment using a pressure cooker in either Tris-EDTA ( $\mathrm{pH}$ 9.0) or citrate buffer ( $\mathrm{pH}$ 6.0). The slides were rinsed in Tis-buffered-saline (TBS) or Phosphate Buffered Saline (PBS) depending on the antibody and blocked with Rodent Block M (Biocare). The primary antibodies CD31, Ki67, smooth muscle $\alpha$-actin ( $\alpha$-SMA), p11, F4/80, were incubated overnight at room temperature followed by secondary antibody and chromogen steps. For anti-rat, the slides were washed with TBS after primary incubation, followed by 15 minutes incubation with rat probe, subsequently the slides were washed and incubated with Rat on mouse HRP polymer for 15 minutes (Biocare), followed by three washes with TBS and stained with DAB (Biocare) and counter stained with hematoxylin. For anti-rabbit secondary antibodies, the slides were incubated with goat anti-rabbit secondary (Envison, DAKO) for 30 minutes, followed by washes with TBS and counterstained with hematoxylin. For anti-mouse antibody, we used mouse-on- mouse HRP polymer kit (Biocare) and anti-goat antibodies we used rabbit-anti goat HRP polymer (DAKO), followed by staining with DAB and counterstaining with hematoxylin. All stained sections were washed in water and mounted with aqueous mounting medium (Vectamount). The immunostained sections were imaged using Zeiss Axio Imager Z1 W/ color and monochrome camera (p11, Ki67, CD31, F4/80, $\alpha$-SMA, H\&E, CD3, and TUNEL staining) using 40X (for manual counting - F4/80, Ki67, CD3) and 20X for representation. To count the number of CD31 positive vessels, we scanned the slides using Aperio Scanning system, following which CD31-positive vessels were counted from 7-10 snapshots of random fields. To quantify number of Ki67 positive, F4/80, CD3-positive, TUNEL positive, we captured images using Zeiss Axio Imager Z1 microscope, and manually counted the positive cells using the Zen software in 7-10 fields. The average of 7-10 fields for Ki67, CD31, F4/80, was used for each mouse in the quantification bar graph. For human p11 staining we 
followed the same procedure as for mouse tissues, but blocking was performed with Background Terminator (Biocare) for 10 minutes before incubating with primary antibody.

\section{Gene expression profiling (mouse tumors)}

Sample preparation, amplification, and hybridization to the Affymetrix Mouse gene 2.0 ST array, and data collection were performed by The Centre for Applied Genomics at the Hospital for Sick Children (Toronto, Canada). The Transcriptome Analysis Console software (ThermoFisher Scientific) was used to normalize data and calculate fold changes in expression (GSE151579). Genes up- or downregulated by more than 2 -fold $\left(\log _{2}=0.678\right)$ at a significance level of $\mathrm{p}<0.05$ were considered differentially expressed.

\section{Gene expression profiling (human tumors)}

The gene expression microarray dataset was generated from a human breast cancer cohort consisting of 176 treatment-naïve primary tumor samples as previously described (58) (GSE22820). Patient material and clinical information were collected under Research Ethics Board Protocol ETH-02-86-17. Patients received standardized guideline-based chemo- and hormone therapies: i.e., hormone therapy for all patients with ER-positive tumors, trastuzumab for those with HER2-overexpression tumors, anthracycline chemotherapy for high risk node-negative disease and anthracycline plus taxane chemotherapy for node-positive disease. The median followup time for surviving patients was 4.5 years.

\section{Quantitative PCR (mouse tumors)}

Tumors from PyMT/p11-WT and PyMT/p11-KO mice at 20 weeks of age were excised, snap frozen in liquid nitrogen, and stored at $-80^{\circ} \mathrm{C}$. Total RNA was extracted using Trizol (Invitrogen) and the PureLink RNA kit (Invitrogen) with DNase treatment. Equal amounts of RNA $(0.5 \mu \mathrm{g})$ were reverse transcribed using iScript (BioRad); quantitative real-time PCR (qPCR) reactions were performed with SsoAdvanced Universal SYBR Supermix (BioRad) and gene-specific mouse primers (Supporting infromation Table S2) on a CFX96 or CFX384 Touch Real-Time PCR Detection system (BioRad). Standard curves for each primer set were generated, and primer efficiencies were incorporated into the CFX Manager software (BioRad). mRNA expression of all samples was calculated using the $\Delta \Delta$ ct method, with gene of interest made relative to two reference genes (rlp10 and PyMT) and an indicated control sample. Relative mRNA expression was $\log -2$ transformed prior to plotting and statistical analysis.

\section{Human patient breast tissue collection, p11 immunohistochemistry and H-score assignment}

Anatomical pathology electronic files (Cerner Millennium) for the Queen Elizabeth II Health Sciences Centre, Nova Scotia Health Authority (NSHA) were retrospectively searched for a cohort of invasive and in situ breast carcinomas beginning January 1, 2011. One formalin-fixed paraffinembedded (FFPE) tumor block and one FFPE block of normal breast tissue were selected from each patient of an identified cohort of 119 patients. For this study, FFPE breast tissues were used with approval from the Nova Scotia Health Authority Research Ethics Board, and Materials Transfer and Collaboration Agreement between the NSHA and Dalhousie University. P11 
immunostaining on patient tissue sections was performed as described earlier (16), using antirabbit polyclonal antibody to human p11 (Proteintech, Rosemont, IL, USA).

The stained sections were evaluated and assigned a semiquantitative score by a pathologist (PJB) in a blinded fashion based on percent of positive cells and intensity of staining. The percentage of cells were identified as negative, weak, moderate, and strong for membrane staining in tumor cells. An $\mathrm{H}$-score of less than $10 \%$ was considered negative. The $\mathrm{H}$-score was determined using the formula ( $\%$ negative $\times 0+\%$ weak $\times 1+\%$ moderate $\times 2+\%$ strong $\times 3)$. The semiquantitative $\mathrm{H}$-score values ranged at 5-250. Nottingham grade 1 and 2 tumors were combined as 'low grade' and Nottingham grade 3 tumors were labeled 'high grade'. A scatter plot was generated based on tumor grade (high and low grade, including samples from the molecular subtype category), tumor type (ductal carcinoma in situ (DCIS), variants of invasive ductal carcinoma and invasive lobular carcinoma, also including samples from the molecular subtype categories), and molecular subtype (estrogen receptor positive $(\mathrm{ER}+)$, Her2+, triple negative $(\mathrm{TN})$.

\section{Isolation of mouse PyMT tumor cell lines}

PyMT tumors from 20-week mice were excised and rinsed in (PBS), minced in RPMI with 5\% FBS with penicillin and streptomycin containing $1 \mathrm{mg} / \mathrm{ml}$ of collagenase (Sigma). The tumor preparation was then incubated at $37^{\circ} \mathrm{C}$ for $1-2$ hours, followed by straining through a 70 -micron mesh strainer. The cells were washed with DMEM with $10 \%$ FBS, passaged once and used in plasmin generation assays as described below. In some cases, isolated cells were directly plated in 96-well plate without passaging and plasmin generation assay was performed as described.

\section{Plasmin generation assay}

Plasmin generation assay was determined in PyMT tumor homogenates. We employed fresh and frozen 20-week old PyMT tumors from PyMT/p11-WT and PyMT/p11-KO mice to prepare tumor homogenates. Briefly the tumors were homogenized in electric homogenizer (Pro Scientific) in Dulbecco's phosphate-buffered saline (DPBS) with 1\% Triton. We used 30-60 $\mu \mathrm{g}$ for the assay. Plasmin generation assay was conducted as described in (44) (19).

\section{Immunoblotting}

Immunoblotting was performed as described in (59) and anti-mouse p11 antibody (R and D systems) and $\beta$-tubulin (Sigma) was used for immune staining (details in Supplemental table 2).

\section{Statistical Analysis}

Three independent experiments with varying mice numbers in each were performed. For evaluation of total palpable tumors (tumor onset), tumor-free mice (survival), tumor growth (volume) and metastasis, we pooled the data/measurements from the three independent experiments. For determination of end-point tumor, and metastatic burden and foci we used data from one independent experiment (20-week endpoint). For time course measurements (whole mounts, tumor progression) we employed 3-12 mice at each endpoint. All statistical analyses were performed using Graph pad prism 5 software (La Jolla, CA, USA). Unless indicated in the figure legends, statistical significance was determined using the Mann Whitney non-parametric test. A significance threshold of $P$ value $<0.05$ was used. 
272 For human patient samples, all statistical analyses for gene expression profiling were performed using MedCalc Version 14.12.0 (MedCalc Software). Gene expression microarray signal intensity values were log-transformed to better fit the normal distribution assumption. One-way ANOVA was employed to test the statistical significance for the difference in S100A10 mRNA levels among molecular subtypes, tumor histologic grades or Ki67 immunoreactivity classifications. Prognostic significance was analyzed using log rank test on Kaplan-Meier survival probabilities.

\section{RESULTS}

\section{Loss of p11 results in delayed appearance of multifocal dysplastic lesions}

The fourth inguinal mammary gland from PyMT/p11-WT and PyMT/p11-KO mice was isolated at 8, 10 and 12 weeks of age, fixed and stained with carmine alum (37). We observed the appearance of early hyperplastic lesion at a single focus or in some cases multiple foci beneath the nipple in the older zone of the ductal tree in the PyMT/p11-WT as early at 6 weeks of age. At 8 weeks, we observed the formation of multiple small nodules in the distal newer ducts, which then spread extensively to the entire length of mammary gland by 12 weeks of age. In contrast, PyMT/p11-KO mice showed complete absence of hyperplastic tumor foci at 6 weeks, which appeared first at 8 weeks of age, and were restricted to only a fraction of the older ductal structures even at 12 weeks. The most dramatic difference was the complete absence of multiple tumor foci throughout the mammary gland of the PyMT/p11-KO mice at 12 weeks (Fig 1).

\section{P11 plays a role in mammary tumor growth and progression}

We employed a cohort of 27 PyMT/p11-WT and 28 PyMT/p11-KO mice and monitored the time of appearance and size of palpable tumors. A fraction of the mice was sacrificed at 20 weeks and the total tumor burden was determined. The remaining mice were monitored further until a combined tumor volume of $4,000 \mathrm{~mm}^{3}$ was attained, the humane endpoint of the experiments.

The first palpable tumor appeared at 9 weeks in the PyMT/p11-WT mice compared to 11 weeks in the PyMT/p11-KO mice (Fig 2A). At 10 weeks 77\% of PyMT/p11-WT and 42\% of PyMT/p11KO mice developed palpable tumors. All the PyMT/p11-WT mice developed palpable tumours by 105 days of age (15 weeks), whereas at 20 weeks only $89 \%$ of PyMT/p11-KO mice developed tumours (Fig 2B).The tumor volume was dramatically decreased in the PyMT/p11-KO mice with a 4 and 6-fold decrease at 15 and 20 weeks respectively (Fig 2D).

Histopathological progression to late carcinoma stage was delayed in PyMT/p11-KO mice (Fig 2E). By 10 weeks of age, $83 \%$ of the PyMT/p11-WT mice and only $25 \%$ of the PyMT/p11-KO had progressed to the early carcinoma stage. By 20 weeks, $82 \%$ of the PyMT/p11-WT mice and $6.25 \%$ of the PyMT/p11-KO had progressed to late carcinoma stage. Interestingly, at this endpoint $37.5 \%$ of PyMT/p11-KO mice showed normal mammary gland histopathology. 
Loss of p11 reduces tumor cell proliferation, vascular density, and macrophage infiltration.

311

312

313

314

315

316

317

318

319

320

321

322

323

324

325

326

327

328

329

330

331

332

333

334

335

336

337

338

339

340

341

342

343

344

345

The proliferative index of PyMT/p11-WT and PyMT/p11-KO tumors at endpoint (20 weeks) were measured using Ki67 positive immunoreactivity (38). We observed a 2.9-fold reduction in mean Ki67 positive-cells in tumors from PyMT/p11-KO mice suggesting that p11 might function in modulation of tumor cell proliferation (Fig 3A). We did not observe a significant difference in tumor cell apoptosis in the PyMT/p11KO mice (Supplemental Fig 2A). We observed a 6-fold reduction in endothelial cell staining (CD31-positive) in the PyMT/p11-KO tumors compared to PyMT/p11-WT tumors (Fig 3B). In both groups of PyMT mice, the macrophages (F4/80-positive) were restricted to the peripheral area of the tumors. However, there was a significant (11.5-fold) decrease in the macrophage density in the tumors from PyMT/p11-KO mice compared to the PyMT/p11-WT mice (Fig 3C). We also observed a qualitative increase in CD3+ (total T cells) staining in the tumors, but this difference was not statistically significant (Supplemental Fig 2B).

\section{Loss of p11 reduced spontaneous and experimental metastasis}

We harvested the lungs from 20 -week old mice and performed histochemical analysis. We observed a significant 18-fold decrease in metastatic burden and a 14-fold decrease in number of metastatic foci (Fig 4A) in the PyMT/p11-KO mice. Furthermore, the presence of metastatic foci was observed only in 3 of 17 (17.6\%) in the PyMT/p11-KO mice compared to 10 of 19 (53\%) in the PyMT/p11-WT mice, suggesting an important role for p11 in spontaneous metastasis. Next, we injected the PyMT transformed cell line, Py8119 with WT p11 levels, intravenously (lateral tail vein) in p11-WT and p11-KO mice. We observed a dramatic decrease in the metastatic burden (6-fold) and number of metastatic foci (2.5-fold) in the p11-KO mice (Fig 4B). This result suggests that stromal p11 is important for the extravasation process and establishment of metastases by these breast cancer cells.

\section{P11 expression is restricted to the stromal compartment in mammary and pulmonary metastatic tumors}

To further investigate the expression and localization of p11 in the PyMT tumor and stromal compartment, we performed immunohistochemical analysis of tumors from both PyMT/p11-WT and PyMT/p11-KO cohorts. Interestingly, we observed that the majority of the p11 staining was localized to the stromal compartment in the PyMT/p11-WT mice. Only a fraction (33\%) of tumors examined presented with diffuse p11 staining in the tumor cells (Fig 5A).

To further evaluate if p11 expression was induced in the metastatic tumor cells, we immunostained lung sections from PyMT/p11-WT mice and observed that the lung tissue but not the metastatic foci showed immunoreactivity for p11. (Fig 5B). This suggested that the tumor cells in the PyMT tumors do not express detectable levels of p11 whereas it is highly expressed in the stromal cells 
surrounding the tumors. Furthermore, there did not appear to be an induction of p11 in the cancer cells that have left the tumor and metastasized to the lungs.

We also examined the lung tumors obtained after tail vein injection of Py8119 cells in p11-WT and p11-KO mice. Surprisingly, we observed that the metastatic foci obtained from the lungs of both genotypes showed strong membranous expression of $\mathrm{p} 11$. This was more evident in the p11$\mathrm{KO}$ mice, where expression was restricted to the small tumor nodules and completely absent in the surrounding lung tissues (Fig 5C). We further evaluated p11 expression in both the whole tumor homogenates and Py8119 cells by immunoblotting (Fig 5D and 5F). As anticipated, whole tumor homogenates from these groups also showed p11 expression in the PyMT/p11-WT tumors consistent with some expression in the stroma. Unlike the PyMT cancer cells, we observed that Py8119 tumor cells showed robust p11 expression (Fig 5F).

\section{Loss of p11 does not affect plasminogen activation (or plasmin generation) in PyMT tumors}

We previously observed that plasminogen activation is substantially reduced in p11-depleted cancer cells such as colorectal (14), fibrosarcoma (13), pancreatic (16), and lung (15). We measured plasmin generation in tumor homogenates and cell lines isolated from PyMT tumors and did not observe any difference (Fig 5E and Supporting Fig 3B). However, PyMT tumors from p11WT and p11-KO mice showed a marked increase in plasminogen activation compared to normal mammary glands (Supplemental Fig 3C). These data suggest that p11 does not play a significant role in tumor cell plasmin generation in the PyMT mammary tumors.

\section{P11 regulates expression of genes and cytokines affecting tumor progression}

To examine how p11 might regulate tumor growth and metastasis genes, we performed microarray gene expression profiling on mammary tumors from PyMT/p11-WT and PyMT/p11-KO mice ( $\mathrm{n}=3$ per group). We observed that 891 transcripts were elevated and 269 were reduced by more than 1.5-fold $(P$ value $<0.05)$ in the PyMT/p11-KO tumors (Fig 6A). As anticipated, S100A10 expression was decreased by 3.08-fold in the PyMT/p11-KO tumors, supporting the validity of the gene expression data set. Of the 891 transcripts, 331 were annotated genes: 144 genes were downregulated and approximately 187 genes were upregulated in the PyMT/p11-KO mice (Supplemental file1). We chose to validate seven downregulated genes (Aldh1a2, Areg, Ctse, Muc1, S100a8, Thbs1, and Tnc) and two upregulated genes (Cdh19 and Cpm) using reverse transcriptase quantitative PCR (RT-qPCR) ( $=11$ mice per group). We confirmed that amphiregulin (Areg), mucin 1 (Muc1), and S100a8, were downregulated whereas cadherin 19 (Cdh 19) was upregulated by qPCR. (Fig 6B). In contrast to the microarray data, Aldh1A2 and Tnc were shown to be upregulated by qPCR (Fig 6B and Supplement Fig 4A).

qPCR showed no significant change in the expression of cytokines associated with macrophage recruitment including Csf1, Ccl5, Ccr5, and Ccl2 (Supplement Fig 4B). Comparison of expression fold), $I l-10$ (3.7-fold), Il-6 (3.7-fold), but no significant changes in Il-12 $\alpha, I l-4$ and Tnf- $\alpha$ (Fig 6C, 
Supporting Fig 4B). Interestingly we observed a 3-fold increase in Csf2 expression in the PyMT/p11-KO tumors (Fig 6C).

\section{P11 is associated with poor clinical outcomes}

We employed a well-defined breast cancer patient cohort $(n=176)$ and stratified the patient population into S100A10 high and low mRNA levels. Kaplan-Meier survival analysis showed that high levels of $S 100 A 10$ were significantly associated with both shorter overall survival $(\mathrm{HR}=3.34$, $\mathrm{p}<0.0001)$ and recurrence-free cancer $(\mathrm{HR}=2.27, \mathrm{p}<0.001)$ (Fig 7A-B). We further found that S100A10 levels were significantly increased in high histologic grade tumors compared with normal mammary tissues $(\mathrm{p}<0.01)$ and low grade $(\mathrm{p}<0.05)$ tumors (Fig 7C) Among the molecular subtypes, S100A10 mRNA levels were significantly higher in TN and HER2-enriched breast tumors compared to normal breast tissues. TN tumors showed significantly higher S100A10 levels than luminal (ER+) tumors (Fig 7D). In addition, tumors with high Ki67 immunoreactivity (percentage of positive cells $>15 \%$ ) showed significantly higher S100A10 levels than tumors with low Ki67 immunoreactivity $(\leq 15 \%)$ (Fig 7E).

Immunostaining of the NSHA patient cohort followed by semiquantitative scoring of the staining showed no p11 immunostaining $(100 \%$ negative, $\mathrm{H}$-score $=0)$ of epithelial cells that formed the normal mammary ducts but observed expression in the stromal areas surrounding the normal duct (Supplement Fig 5A). Based on H-score, we found 25\% of low grade (LG), 29.3\% of high grade (HG), $37.5 \%$ of DCIS, $33 \%$ of IDC, $23.8 \%$ of ER+, 37.5\% Her2+ and $52 \%$ of TN tissues/samples were positive for $\mathrm{p} 11$ expression. We observed a significant increase in H-score values between normal and LG, and normal and HG, but the difference between LG and HG was not significant (Fig 7F). Similarly, DCIS and IDC tissues/samples/patients showed significantly higher H-score values compared to normal tissues. But there was no difference in p11 expression between DCIS and IDC patients, suggesting that p11 expression does not correlate with invasive progression (Fig $7 \mathrm{G}$ and supplemental Fig 5). We observed the most dramatic increase in p11 staining between normal tissue and TN tumors ( $\mathrm{p}$-value $<0.0001)$, consistent with the highest mean $\mathrm{H}$-score value for the TN tumors. We also noted significant increase between normal and ER+, and normal and Her2+ tumors, but there was no significant increase between ER+ and Her2+ tumors. Interestingly, statistical comparison of ER+ and TN tumors showed increase in TN with respect to ER+ although the $P$ value (0.0511) was only approaching significance (Fig $7 \mathrm{H}$ and supplemental Fig 5).

\section{Discussion}

The loss of components of the plasmin-plasminogen system decreased pulmonary metastasis but had no effect on tumor onset or growth in the PyMT model (reviewed in (4)). Interestingly, in this study we observed a dramatic delay in tumor onset, growth, and progression to malignancy in the PyMT/p11KO mice. These results suggest a complex role for p11 in breast cancer oncogenesis beyond the regulation of plasmin generation. 
We observed a dramatic decrease in metastatic burden (18-fold) and metastatic foci (14-fold) in PyMT/p11-KO mice. The histopathological progression to late carcinoma stage was delayed in PyMT/p11KO mice (Fig 2E), potentially attributing the decrease in metastases to the delayed development of malignancy. However, pulmonary metastasis in the PyMT model is an early event and independent of tumor size $(39,40)$. Interestingly, metastases formed by injection of the PyMT transformed cell line, Py8119, into the p11-KO mice were dramatically reduced, suggesting that the p11-deficient stroma is a less favorable environment for the establishment of metastases.

Our results are consistent with our previous work showing that the growth of Lewis lung carcinomas or T241 fibrosarcomas were dramatically reduced in p11-deficient mice compared with wild-type mice (19) and that the tumor growth deficit corresponded with a decrease in macrophage and endothelial cell density. Tumor-associated macrophages (TAMs) significantly contribute to tumor progression, angiogenic switch, and metastasis $(41,42)$, with loss of peritumoral TAMs resulting in delayed tumor progression (43). Therefore, it is likely that a portion of the requirement of p11 for PyMT-driven tumor growth and metastasis is due to the function of p11 in macrophage recruitment.

We have previously reported that p11 is responsible for majority of the plasmin generation in various cancer cell lines $(5,15,16,44)$. However, in the present study we were unable to detect any differences in plasmin generation between cancer cells or tumor homogenates isolated from the PyMT/p11-KO and PyMT/p11-WT mice. However, we observed that PyMT tumors from p11WT and p11-KO mice showed a marked increase in plasmin generation compared to normal mammary glands (Supplemental Fig 3D), suggesting compensation by other plasminogen receptors in this model. It is likely that distant peripheral macrophages utilize plasminogen receptors such as p11 to migrate and invade the tumors $(19,45,46)$. Furthermore, we detected weak p11 expression in some of the tumor cells and no expression in metastatic nodules of PyMT/p11WT tumors. In contrast, lung tumors obtained after tail vein injection of Py8119 cells in p11-WT and p11-KO mice showed robust p11 expression. Overall, the exclusive expression of p11 in the stroma in vivo parallels the stromal localization of other components of the plasminogen activation system including UPA, uPAR, tPA and PAI-1 in mouse (PyMT) and human models (4). The lack of p11 expression in the PyMT-expressing tumor cells and restriction of the majority of p11 expression to the stromal compartment were striking observations in the current study. The middle T (MT) region of PyMT is an effective oncogene and activates the PI3 kinase signaling pathway $(47,48)$. We recently showed that active PI3K signaling decreased p11 expression in several tumor cells via the FOXC2 transcription factor (15). In addition in vitro observations indicate a distinct rewiring of signaling in 2-D cell cultures resulting in increased p11 expression potentially due to derepression of tumor-stroma signaling events observed in vivo $(49,50)$. Furthermore, the retention of p11 expression in the Py8119 tumor cells in the lung metastatic nodules, suggests the absence of inhibitory signals in the lung microenvironment.

459 Whole mount analysis of mammary glands indicated a decrease in multifocal dysplastic lesions as 460 early as 6-8 weeks in the PyMT/p11-KO mice (Fig 1). An interesting observation was the 461 reduction in length of the ductal branches in the PyMT/p11-KO mammary glands at the early time 462 points of 6 and 8 weeks, and was comparable to the WT at 10 and 12 weeks (Supplemental Fig 1). 
Other groups have shown that infiltration of macrophages is required for early mammary gland development (51). It is likely that defective macrophage migration observed with p11-KO mice causes early defects in ductal branching in the mammary gland. This suggests that p11 may play a potential role during early stages of mammary gland development. More recently, another plasminogen receptor, Plg-Rkt, was shown to be critical for lactogenesis and mammary lobuloalveolar development (52).

qPCR validation of microarray gene data confirmed the significant downregulation of AREG, MUC1, and S100A8 genes in PyMT/p11-KO tumors. AREG (amphiregulin), a player in breast cancer proliferation, is abundant in the pubertal mammary gland and loss of AREG in mice results in stunted ductal morphogenesis. Our observation of decreased early mammary ductal morphogenesis might be due to the loss of AREG expression (53). Loss of transmembrane glycoprotein MUC1 results in significant delay in tumor progression and metastasis (54), consistent with delayed progression to malignancy displayed by PyMT/p11-KO tumors. S100A8 is elevated in ER- and Her2+ subtypes of breast cancer and contributes towards cancer cell survival and metastasis (55), thereby contributing to delayed progression of PyMT/p11-KO tumors. It is unclear whether 11 plays a direct or indirect role in regulating these genes in vivo, or whether the alteration of these genes are markers of tumor growth and progression.

We also compared the levels of inflammatory modulators in PyMT/p11WT and PyMT/p11-KO tumors that might impact macrophage infiltration. The CSF-1 gene, implicated in the proliferation, differentiation and recruitment of macrophages in breast cancer (37) was unchanged. We saw a significant increase in CSF-2 expression in PyMT/p11-KO tumors but CSF-2 has not been shown to correlate with macrophage infiltration in breast tumors. We also observed a significant increase in cytokines such as Ifn- $\gamma, \mathrm{Il}-6$ and $\mathrm{Il}-10$. Il-6 positively correlates with breast cancer progression and development of metastasis (56). Studies on the role of $I l-10$ in breast cancer showed contradicting results (56). If $n-\gamma$ plays an important role in antitumor immunity and tumor suppressive phenotype via the JAK/STAT pathway. It is possible that the tumor suppressive phenotype observed in the PyMT/p11-KO mice is ascribed to the increase in tumor suppressive cytokines such as $I l-10$ and $I f n-\gamma$.

Previous gene expression studies showed that p11 correlated to poor overall survival in basal-like breast cancer (reviewed in (57). In elucidating the prognostic role of p11 in human breast cancer, we found that p11 mRNA was overexpressed in human breast tumors, correlated positively with overall survival and recurrence-free survival, and was increased in high grade tumors compared to low grade tumors and normal tissue. P11 was highly expressed in TN breast cancer, ER+ and HER2+ suggesting a role in breast cancer progression. Interestingly, expression of p11 protein was significantly elevated in tumor cells compared to normal mammary epithelium, but we did not observe any correlation between high p11 expression and clinical and pathological tumor grade or with molecular subtype in human breast cancer samples. The lack of consistency between our gene expression profiling and immunohistochemistry data could be due to two reasons. First, microarray analysis was performed in tumors containing stroma making the increased p11 expression a contribution of the tumor microenvironment. Second, we conducted p11 immunostaining only in a small cohort of patient samples with a small sample size for ER+ and HER2+ tumors. Future studies will be aimed at increasing the sample size for IHC or by using a Tissue Microarray 
analysis and evaluating the expression pattern in the stroma. Overall p11 expression was completely restricted to the stromal cells in the PyMT mouse model, whereas expression was seen in human tumor cells. This difference can potentially be attributed to the nature of oncogene and mutations between the murine and human breast tumors.

\section{Conclusion}

Our current study is the first comprehensive study using a transgenic mouse model to examine the role of p11 in breast cancer. These results demonstrated that p11 plays a causal, complex, and definitive role in breast tumor development, progression, and metastasis, possibly via p11dependent macrophage migration and tumor infiltration. Surprisingly, our results suggest that stromal and not cancer cell p11 is crucial for mediating these effects specifically in the PyMT model. Since the majority of p11 was expressed by the stromal cells in the tumor periphery, our study also highlights the importance of tumor-stroma interactions and signaling for breast tumor progression. Further studies are required to conclusively demonstrate that p11 can be employed as a biomarker with diagnostic and prognostic value in breast cancer.

\section{Availability of data and materials}

All data collected has been presented in the manuscript and supporting information and is available upon request.

\section{Acknowledgements}

The authors would like to thank Allison Glover, Eva Rogerson, Erica Meehan, Allison Letcher for their technical support. We would also like to thank Victoria Miller for their thoughtful comments and discussions.

\section{Funding}

AGB is supported by a trainee award from the Beatrice Hunter Cancer Research Institute with funds provided by the Harvey Graham Cancer Research Fund as part of The Terry Fox Strategic Health Research Training Program in Cancer Research at CIHR. MLD is supported by CGS-D award from the CIHR, a Nova Scotia Health Research Foundation studentship, a Nova Scotia Research and Innovation Graduate scholarship, a Beatrice Hunter Cancer Research Institute Cancer Research Training Program studentship, and a Killam Laureate scholarship. Paola A Marignani is supported by funding from Dalhousie Medical Research Foundation (DMRF) and Breast Cancer Society of Canada (BCSC). Rosaline Godbout is supported by a grant from Canadian Cancer Research Institute (grant No. 705455). Catherine KL Too is supported Canadian Breast Cancer Foundation/Atlantic, Canadian Cancer Society and Breast Cancer Society of Canada, QEII Foundation, Beatrice Hunter Cancer Research Institute, Penelope J. Barnes is supported by Breast Cancer Society of Canada, QEII Foundation, Beatrice Hunter Cancer Research Institute. Paola Marcato is supported by CIHR, PJT 162313. David M Waisman and this research is supported by a grant from the CIHR. 


\section{Affiliations}

$543{ }^{1}$ Department of Pathology, Dalhousie University, Halifax, Nova Scotia, Canada

544 Alamelu G. Bharadwaj, Margaret L. Dahn, Patricia Colp, Penelope J. Barnes, Paola Marcato, 545 David M. Waisman

$546{ }^{2}$ Department of Biochemistry \& Molecular Biology, Dalhousie University, Halifax, Nova Scotia, 547 Canada

548 Lynn N. Thomas, Ryan W. Holloway, Paola A. Marignani, Catherine KL. Too, David M.

549 Waisman

$550{ }^{3}$ Department of Microbiology and Immunology, Dalhousie University, Halifax, Nova Scotia, 551 Canada

552 Paola Marcato

$553{ }^{4}$ Department of Oncology, University of Alberta, Edmonton, Alberta, Canada

554 Ronzong Liu, Rosaline Godbout

555 Contributions

556 Conceptualization: AGB and DMW. Methodology: AGB, MLD, RZL, PC, RWH, LNT, PAM, 557 CKLT, PJB, RG, PM and DMW. Formal analysis: AGB, MLD, RZL and DMW. Investigation: 558 AGB, MLD, RZL and DMW. Writing: AGB, MLD, RZL and DMW. Supervision: DMW. 559 Funding acquisition: DMW, PAM, CKLT, PJB, RG, PM. The author(s) read and approved the 560 final manuscript.

561 Corresponding author - David M Waisman

562 Ethics declarations

563 All animal experiments were performed according to protocol approved by University 564 Committee on Laboratory Animals, Dalhousie University, Canada.

565 Ethics approval and consent to participate

566 Patient material and clinical information were collected under Research Ethics Board Protocol 567 ETH-02-86-17.

568 Consent for publication

569 Not applicable 


\section{Competing interests}

571 The authors declare that they have no conflicts of interest with the contents of this article.

572

573 References

574 1. Lambert AW, Pattabiraman DR, Weinberg RA. Emerging Biological Principles of $575 \quad$ Metastasis. Cell. 2017 09;168(4):670-91.

576

577

578

579

580

581

582

583

584

585

586

587

588

589

590

591

592

593

594

595

596

597

598

599

600

601

602

2. Danø K, Behrendt N, Høyer-Hansen G, Johnsen M, Lund LR, Ploug M, et al. Plasminogen activation and cancer. Thromb Haemost. 2005 Apr;93(4):676-81.

3. Sevenich L, Joyce JA. Pericellular proteolysis in cancer. Genes Dev. 2014 Nov $1 ; 28(21): 2331-47$.

4. Almholt K, Green KA, Juncker-Jensen A, Nielsen BS, Lund LR, Rømer J. Extracellular proteolysis in transgenic mouse models of breast cancer. J Mammary Gland Biol Neoplasia. 2007 Mar;12(1):83-97.

5. Madureira PA, O'Connell PA, Surette AP, Miller VA, Waisman DM. The biochemistry and regulation of S100A10: a multifunctional plasminogen receptor involved in oncogenesis. J Biomed Biotechnol. 2012;2012:353687.

6. Kwon M, MacLeod TJ, Zhang Y, Waisman DM. S100A10, annexin A2, and annexin a2 heterotetramer as candidate plasminogen receptors. Front Biosci J Virtual Libr. 2005 Jan $1 ; 10: 300-25$.

7. Kassam G, Le BH, Choi KS, Kang HM, Fitzpatrick SL, Louie P, et al. The p11 subunit of the annexin II tetramer plays a key role in the stimulation of t-PA-dependent plasminogen activation. Biochemistry. 1998 Dec 1;37(48):16958-66.

8. Kassam G, Choi KS, Ghuman J, Kang HM, Fitzpatrick SL, Zackson T, et al. The role of annexin II tetramer in the activation of plasminogen. J Biol Chem. 1998 Feb 20;273(8):4790-9.

9. Miller VA, Madureira PA, Kamaludin AA, Komar J, Sharma V, Sahni G, et al. Mechanism of plasmin generation by S100A10. Thromb Haemost. 2017 02;117(6):1058-71.

10. MacLeod TJ, Kwon M, Filipenko NR, Waisman DM. Phospholipid-associated annexin A2S100A10 heterotetramer and its subunits: characterization of the interaction with tissue plasminogen activator, plasminogen, and plasmin. J Biol Chem. 2003 Jul 11;278(28):2557784.

11. Bharadwaj A, Bydoun M, Holloway R, Waisman D. Annexin A2 heterotetramer: structure and function. Int J Mol Sci. 2013 Mar 19;14(3):6259-305. 
12. Noye TM, Lokman NA, Oehler MK, Ricciardelli C. S100A10 and Cancer Hallmarks: Structure, Functions, and its Emerging Role in Ovarian Cancer. Int J Mol Sci. 2018 Dec $19 ; 19(12)$.

13. Choi K-S, Fogg DK, Yoon C-S, Waisman DM. p11 regulates extracellular plasmin production and invasiveness of HT1080 fibrosarcoma cells. FASEB J Off Publ Fed Am Soc Exp Biol. 2003 Feb;17(2):235-46.

14. Zhang L, Fogg DK, Waisman DM. RNA interference-mediated silencing of the S100A10 gene attenuates plasmin generation and invasiveness of Colo 222 colorectal cancer cells. J Biol Chem. 2004 Jan 16;279(3):2053-62.

15. Bydoun M, Sterea A, Weaver ICG, Bharadwaj AD, Waisman DM. A novel mechanism of plasminogen activation in epithelial and mesenchymal cells. Sci Rep. 2018 20;8(1):14091.

16. Bydoun M, Sterea A, Liptay H, Uzans A, Huang W-Y, Rodrigues GJ, et al. S100A10, a novel biomarker in pancreatic ductal adenocarcinoma. Mol Oncol. 2018 Nov;12(11):1895916.

17. O'Connell PA, Surette AP, Liwski RS, Svenningsson P, Waisman DM. S100A10 regulates plasminogen-dependent macrophage invasion. Blood. 2010 Aug 19;116(7):1136-46.

18. Surette AP, Madureira PA, Phipps KD, Miller VA, Svenningsson P, Waisman DM. Regulation of fibrinolysis by S100A10 in vivo. Blood. 2011 Sep 15;118(11):3172-81.

19. Phipps KD, Surette AP, O'Connell PA, Waisman DM. Plasminogen receptor S100A10 is essential for the migration of tumor-promoting macrophages into tumor sites. Cancer Res. 2011 Nov 1;71(21):6676-83.

20. Sato K, Saiki Y, Arai K, Ishizawa K, Fukushige S, Aoki K, et al. S100A10 upregulation associates with poor prognosis in lung squamous cell carcinoma. Biochem Biophys Res Commun. 2018 Oct 28;505(2):466-70.

21. Katono K, Sato Y, Jiang S-X, Kobayashi M, Saito K, Nagashio R, et al. Clinicopathological Significance of S100A10 Expression in Lung Adenocarcinomas. Asian Pac J Cancer Prev APJCP. 2016;17(1):289-94.

22. Gocheva V, Naba A, Bhutkar A, Guardia T, Miller KM, Li CM-C, et al. Quantitative proteomics identify Tenascin-C as a promoter of lung cancer progression and contributor to a signature prognostic of patient survival. Proc Natl Acad Sci U S A. 2017 11;114(28):E562534 .

23. McKiernan E, McDermott EW, Evoy D, Crown J, Duffy MJ. The role of S100 genes in breast cancer progression. Tumour Biol J Int Soc Oncodevelopmental Biol Med. 2011 Jun;32(3):441-50. 
24. Yu M, Bardia A, Wittner BS, Stott SL, Smas ME, Ting DT, et al. Circulating breast tumor cells exhibit dynamic changes in epithelial and mesenchymal composition. Science. 2013 Feb 1;339(6119):580-4.

25. Lin EY, Jones JG, Li P, Zhu L, Whitney KD, Muller WJ, et al. Progression to malignancy in the polyoma middle $\mathrm{T}$ oncoprotein mouse breast cancer model provides a reliable model for human diseases. Am J Pathol. 2003 Nov;163(5):2113-26.

26. Maglione JE, Moghanaki D, Young LJ, Manner CK, Ellies LG, Joseph SO, et al. Transgenic Polyoma middle-T mice model premalignant mammary disease. Cancer Res. 2001 Nov 15;61(22):8298-305.

27. Fantozzi A, Christofori G. Mouse models of breast cancer metastasis. Breast Cancer Res BCR. 2006;8(4):212.

28. Bugge TH, Lund LR, Kombrinck KK, Nielsen BS, Holmbäck K, Drew AF, et al. Reduced metastasis of Polyoma virus middle $\mathrm{T}$ antigen-induced mammary cancer in plasminogendeficient mice. Oncogene. 1998 Jun 18;16(24):3097-104.

29. Almholt K, Lærum OD, Nielsen BS, Lund IK, Lund LR, Rømer J, et al. Spontaneous lung and lymph node metastasis in transgenic breast cancer is independent of the urokinase receptor uPAR. Clin Exp Metastasis. 2015 Aug;32(6):543-54.

30. Almholt K, Lund LR, Rygaard J, Nielsen BS, Danø K, Rømer J, et al. Reduced metastasis of transgenic mammary cancer in urokinase-deficient mice. Int J Cancer. 2005 Feb $10 ; 113(4): 525-32$.

31. Almholt K, Juncker-Jensen A, Laerum OD, Danø K, Johnsen M, Lund LR, et al. Metastasis is strongly reduced by the matrix metalloproteinase inhibitor Galardin in the MMTV-PymT transgenic breast cancer model. Mol Cancer Ther. 2008 Sep;7(9):2758-67.

32. Juncker-Jensen A, Rømer J, Pennington CJ, Lund LR, Almholt K. Spontaneous metastasis in matrix metalloproteinase 3-deficient mice. Mol Carcinog. 2009 Jul;48(7):618-25.

33. Sevenich L, Werner F, Gajda M, Schurigt U, Sieber C, Müller S, et al. Transgenic expression of human cathepsin B promotes progression and metastasis of polyoma-middle-T-induced breast cancer in mice. Oncogene. 2011 Jan 6;30(1):54-64.

34. Vasiljeva O, Korovin M, Gajda M, Brodoefel H, Bojic L, Krüger A, et al. Reduced tumour cell proliferation and delayed development of high-grade mammary carcinomas in cathepsin B-deficient mice. Oncogene. 2008 Jul 10;27(30):4191-9.

35. Ricciardelli C, Frewin KM, Tan I de A, Williams ED, Opeskin K, Pritchard MA, et al. The ADAMTS1 protease gene is required for mammary tumor growth and metastasis. Am J Pathol. 2011 Dec;179(6):3075-85. 
36. Wyckoff J, Wang W, Lin EY, Wang Y, Pixley F, Stanley ER, et al. A paracrine loop between tumor cells and macrophages is required for tumor cell migration in mammary tumors. Cancer Res. 2004 Oct 1;64(19):7022-9.

37. Lin EY, Nguyen AV, Russell RG, Pollard JW. Colony-stimulating factor 1 promotes progression of mammary tumors to malignancy. J Exp Med. 2001 Mar 19;193(6):727-40.

38. Scholzen T, Gerdes J. The Ki-67 protein: from the known and the unknown. J Cell Physiol. 2000 Mar;182(3):311-22.

39. Hüsemann Y, Geigl JB, Schubert F, Musiani P, Meyer M, Burghart E, et al. Systemic spread is an early step in breast cancer. Cancer Cell. 2008 Jan;13(1):58-68.

40. Weng D, Penzner JH, Song B, Koido S, Calderwood SK, Gong J. Metastasis is an early event in mouse mammary carcinomas and is associated with cells bearing stem cell markers. Breast Cancer Res BCR. 2012 Jan 25;14(1):R18.

41. Pollard JW. Macrophages define the invasive microenvironment in breast cancer. J Leukoc Biol. 2008 Sep;84(3):623-30.

42. Pollard JW. Tumour-educated macrophages promote tumour progression and metastasis. Nat Rev Cancer. 2004;4(1):71-8.

43. Rumney RMH, Coffelt SB, Neale TA, Dhayade S, Tozer GM, Miller G. PyMT-Maclow: A novel, inducible, murine model for determining the role of CD68 positive cells in breast tumor development. PloS One. 2017;12(12):e0188591.

44. Madureira PA, Bharadwaj AG, Bydoun M, Garant K, O'Connell P, Lee P, et al. Cell surface protease activation during RAS transformation: Critical role of the plasminogen receptor, S100A10. Oncotarget. 2016 26;7(30):47720-37.

45. Das R, Burke T, Plow EF. Histone H2B as a functionally important plasminogen receptor on macrophages. Blood. 2007 Nov 15;110(10):3763-72.

46. Miles LA, Lighvani S, Baik N, Khaldoyanidi S, Mueller BM, Parmer RJ. New Insights into the Role of Plg-RKT in Macrophage Recruitment. Int Rev Cell Mol Biol. 2014;309:259302.

47. Ichaso N, Dilworth SM. Cell transformation by the middle T-antigen of polyoma virus. Oncogene. 2001 Nov 26;20(54):7908-16.

48. Denis D, Rouleau C, Schaffhausen BS. A Transformation-Defective Polyomavirus Middle T Antigen with a Novel Defect in PI3 Kinase Signaling. J Virol. 2017 Jan 15;91(2).

49. Riedl A, Schlederer M, Pudelko K, Stadler M, Walter S, Unterleuthner D, et al. Comparison of cancer cells in 2D vs 3D culture reveals differences in AKT-mTOR-S6K signaling and drug responses. J Cell Sci. 2017 01;130(1):203-18. 
50. Weigelt B, Lo AT, Park CC, Gray JW, Bissell MJ. HER2 signaling pathway activation and response of breast cancer cells to HER2-targeting agents is dependent strongly on the 3D microenvironment. Breast Cancer Res Treat. 2010 Jul;122(1):35-43.

51. Van Nguyen A, Pollard JW. Colony stimulating factor-1 is required to recruit macrophages into the mammary gland to facilitate mammary ductal outgrowth. Dev Biol. 2002 Jul $1 ; 247(1): 11-25$.

52. Miles LA, Baik N, Bai H, Makarenkova HP, Kiosses WB, Krajewski S, et al. The plasminogen receptor, Plg-RKT, is essential for mammary lobuloalveolar development and lactation. J Thromb Haemost JTH. 2018;16(5):919-32.

53. McBryan J, Howlin J, Napoletano S, Martin F. Amphiregulin: role in mammary gland development and breast cancer. J Mammary Gland Biol Neoplasia. 2008 Jun;13(2):159-69.

54. Nath S, Mukherjee P. MUC1: a multifaceted oncoprotein with a key role in cancer progression. Trends Mol Med. 2014 Jun;20(6):332-42.

55. Lim SY, Yuzhalin AE, Gordon-Weeks AN, Muschel RJ. Tumor-infiltrating monocytes/macrophages promote tumor invasion and migration by upregulating S100A8 and S100A9 expression in cancer cells. Oncogene. 2016 03;35(44):5735-45.

56. Méndez-García LA, Nava-Castro KE, Ochoa-Mercado T de L, Palacios-Arreola MI, RuizManzano RA, Segovia-Mendoza M, et al. Breast Cancer Metastasis: Are Cytokines Important Players During Its Development and Progression? J Interferon Cytokine Res Off J Int Soc Interferon Cytokine Res. 2019;39(1):39-55.

57. Zhang S, Wang Z, Liu W, Lei R, Shan J, Li L, et al. Distinct prognostic values of S100 mRNA expression in breast cancer. Sci Rep. 2017 04;7:39786.

58. Liu R-Z, Graham K, Glubrecht DD, Germain DR, Mackey JR, Godbout R. Association of FABP5 expression with poor survival in triple-negative breast cancer: implication for retinoic acid therapy. Am J Pathol. 2011 Mar;178(3):997-1008.

59. Holloway RW, Thomas ML, Cohen AM, Bharadwaj AG, Rahman M, Marcato P, et al. Regulation of cell surface protease receptor S100A10 by retinoic acid therapy in acute promyelocytic leukemia (APL)汿. Cell Death Dis. 2018 Sep 11;9(9):920.

\section{Figure Legends}

Fig 1: Loss of p11 affects formation of hyperplastic lesions in PyMT driven tumors. Whole mammary glands from PyMT/p11-WT (n=3) and PyMT/p11-KO mice at (8, 10, 12 weeks) were excised, fixed, and stained with Carmine Alum as per standard protocols (See Materials and Methods). Representative images from the $4^{\text {th }}$ abdominal mammary gland from each group and time point is shown. 

PyMT/p11-KO mice. (A) Appearance of palpable tumors was monitored weekly. (B) Percent tumor-free mice was calculated using Kaplan-Meir analysis (Hazard ration -2.24 , $P$ value 0.0004). (C) Tumor volume was measured weekly using calipers. Total volume was plotted to represent tumor growth rate. (D) Total tumor weight at endpoint (20 weeks) was determined as a percentage of body weight. Data is a compilation of three independent experiments, except (D) which is data from one independent experiment. Significance was determined by non-parametric t-test (Mann Whitney U test - unpaired, non-parametric). (E) Mammary glands and tumors from PyMT/p11-WT and PyMT/p11-KO mice were harvested at 20 weeks, formalin-fixed, embedded, and sectioned at $5 \mu \mathrm{m}$ thickness. The H\&E and smooth muscle $\alpha$-actin-stained sections were classified into histopathological stages by a pathologist in a blinded manner. ( $n=6$ to 12 mice in each group). Statistical analysis was performed by $\chi^{2}$ - test $(P$ value $<0.0001)$.

Fig 3: PyMT/p11-KO mice tumors show reduced proliferation vascular density and macrophage infiltration. Formalin-fixed, paraffin-embedded and sectioned tissues from PyMT/p11-WT $(n=11)$ and PyMT/p11-KO (n=10) (end-point, 20 weeks) mice were immunostained for (A) Ki67 (proliferation marker), (B) CD31 (endothelial marker) and (C) F4/80 (mouse macrophage marker). (A) Immunstaining using anti-rabbit Ki67 antibody (Abcam). Stained sections were imaged using Zeiss Zeiss Axio Imager Z1 W/color and monochrome camera at 10X magnification. Left panel: The number of Ki67-positive cells was manually counted using Zen (2012) software in 7-10 random fields per tissue section/mouse. Significance was determined using Mann Whitney U test (unpaired, non-parametric), $P$ value is 0.0021 . Right panel: The representative image was captured at $20 \mathrm{X}$ magnification. Scale bar $-100 \mu \mathrm{m}$. (B) Immunostaining using anti-rabbit CD31 antibody (Abcam). Stained sections were imaged using Aperio Scanning system (Leica Biosystems, Concord, Ontario) at 40X magnification. Left panel: The number of CD31-positive cells were manually counted using Imagescope software in 7-10 random fields per tissue section/mouse. Mann Whitney U test (unpaired, non-parametric) shows $P$ value $<0.0001$. Right panel: Representative image at $20 \mathrm{X}$ magnification. Scale bar - $100 \mu \mathrm{m}$. (C) Immunostaining using anti-rat F4/80 antibody (BM8, Thermofisher Scientific). Stained sections were imaged as in (A), but at 40X magnification. Left panel: The number of F4/80-positive cells were manually counted as in (A). Mann Whitney U test (unpaired, non-parametric) shows $P$ value $<0.0001$. Right panel: Representative image at 20X magnification. Scale bar - $100 \mu \mathrm{m}$.

Fig 4: Metastasis is diminished in PyMT/p11-KO mice. (A) We evaluated pulmonary metastasis at 20-week end point in (spontaneous model) PyMT/p11-WT and PyMT/p11-KO mice by microscopic examination of formalin-fixed, H\&E-stained lung sections $(5 \mu \mathrm{m})$. Three lung sections with each $100 \mu \mathrm{m}$ apart were used for staining. Quantification was performed by Aperio image analysis software (Imagescope). Mean values for three sections were used to calculate the metastatic burden and foci values. (A) Metastatic burden - calculated the total metastatic area and normalized to total lung area (A- upper left panel). Mann Whitney U test show statistical significance with $P$ value of 0.0062 . The number of metastatic foci per mouse lung section was determined by manual counting of imaged sections (A- upper right panel). Mann Whitney U test show $P$ value of 0.0069. Lower panels: representative lung images from WT and KO mice. (B) Experimental metastasis assay. We injected $2.5 \times 10^{5}$ Py8119 (p11-WT levels) cells into p11-WT 
and $\mathrm{p} 11-\mathrm{KO}$ mice ( $\mathrm{n}=12$ mice per group). The lungs were harvested after 14 days, formalin-fixed, and sectioned at $5 \mu \mathrm{m}$ as described above. Mann Whitney non-parametric t- test for statistical significance was performed. Metastasis was pooled and combined from two independent experiments (n=6 each). $P$ value $<0.0001$. Lower panels: representative lung images from WT and $\mathrm{KO}$ mice.

787

788

789

790

791

792

793

794

795

796

797

798

799

800

801

802

803

804

805

806

807

808

809

810

811

812

813

814

815

816

817

818

819

820

821

822

823

Fig 5: Expression of p11 in mammary and pulmonary metastatic tumors is restricted to the stromal compartment in the PyMT/p11-WT tumors. Immunohistochemical staining was performed using anti-rabbit p11 antibody (Proteintech), on $5 \mu \mathrm{m}$ sections from 20 -week endpoint PyMT/p11-WT (n=10) and PyMT/p11-KO tumors $(n=3)$, and lungs from spontaneous and experimental metastasis assay. (A) Representative images of $\mathrm{p} 11$ immunostained sections of PyMT mammary tumors. As anticipated the tumors from PyMT/p11-KO mice showed no staining, validating the specificity of the antibody. (B) PyMT spontaneous lung metastasis from PyMT/p11-WT and PyMT/p11-KO mice, and (C) experimental metastasis of Py8119 cells injected in p11-WT $(n=4)$ and p11-KO $(n=4)$ mice are shown. Scale bar is $100 \mu \mathrm{m}$. (D) Western blot of p11 expression in total cell tumor homogenates from PyMT/p11-WT $(n=3)$ and PyMT/p11-KO mice $(n=3)$. (E) Fresh and frozen tumors from both PyMT/p11-WT ( $\mathrm{n}=6$ mice) and PyMT/p11-KO ( $\mathrm{n}=5$ mice) were homogenized and equal protein $(30-60 \mu \mathrm{g})$ were used for plasmin generation assay as described in Materials and Methods and supplemental section. Mann Whitney U (unpaired, non-parametric) t- test show P value of 0.3290). NS, not significant (F) Western blot for p11 expression in total cell lysates in Py8119 cells obtained from ATCC $(\mathrm{n}=4)$ using goat anti-mouse antibody ( $\mathrm{R}$ and $\mathrm{D}$ systems).

Fig 6: Tumor transcriptome of PyMT/p11-KO mice suggest the downregulation of tumor promoting genes. Gene expression profiling of PyMT/p11-WT and PyMT/p11-KO tumors were performed ( $\mathrm{n}=3$ mice per group). (A) Volcano plot of significantly $P$ value $<0.05$ ) upregulated and downregulated genes in the PyMT/p11 KO mice ( $n=3$ mice in each group). The top 6 genes are highlighted. Genes which were up- or down-regulated more than 2-fold $\left(\log _{2}=0.678\right)$ at a significance level of $P$ value $<0.05$ were considered differentially expressed. (B) Validation of the top 9 differentially expressed genes using quantitative RT-PCR (10-11 mice per group). Significance was determined by unpaired t- test. Only the genes significantly altered are shown. (C) Loss of p11 in PyMT tumors results in differential cytokine expression profile. We performed quantitative RT-PCR on tumors isolated from PyMT/p11-WT and PyMT/p11KO mice (10-11 mice per group), using a CFX96 or CFX384 Touch Real-Time PCR Detection system (BioRad). Relative mRNA expression was log-2 transformed prior to plotting and statistical analysis. Significance was determined by unpaired, t- test. Only those cytokines significantly altered are shown.

Fig 7: Expression of S100A10 (p11) expression in breast cancer patients. Treatment-naïve primary breast cancer samples $(n=176)$ were obtained through the $\mathrm{CBCF}$ Tumor Bank and used for gene expression analysis. S100A10 high and low mRNA levels were based on the signal intensity from our gene expression microarray profile using the Receiver Operating Characteristic (ROC) curve analysis. Correlation of S100A10 (p11) mRNA with (A) overall survival probability. High mRNA levels of p11 gene (p11) are significantly associated with poor patient overall survival 
824 (hazard ratio of 3.34). (B) Recurrence free survival, with hazard ratio 2.27. (C) For histological 825 tumor grade, $\mathrm{p} 11$ is significantly upregulated in high grade tumors compared to normal breast and 826 low-grade tumor tissues. (D) For molecular subtype, p11 is significantly up-regulated in HER2+ 827 and triple negative breast cancers compared to normal breast tissues. (E) In breast cancer patients, 828 p11 mRNA levels are significantly higher in tumors with high Ki67 immunoreactivity. S100A10 829 (p11) protein is overexpressed in breast tumors compared to normal mammary tissues. (F, $830 \mathrm{G}, \mathrm{H})$ Immunohistochemical staining of p11 in normal breast tissues and in low grade (LG), high 831 grade (HG), ductal carcinoma in situ (DCIS), IDC/Invasive, ER+, Her2+ and Triple negative (TN) 832 tumors was performed using anti-p11 antibody (Proteintech). These human tissues were obtained 833 from Queen Elizabeth II Health Sciences Centre, Nova Scotia Health Authority (see Materials and 834 Methods). The stained sections were scored (semi-quantitative) based on percent positive tumor 835 cells and intensity of staining by a pathologist (blinded). $\mathrm{H}$ score was determined based on the 836 formula described and the samples/tissues were separated and plotted based on (F) 837 clinical/histological grade, $(\mathrm{G})$ pathological grade, and (H) molecular subtype. Statistical analysis 838 was performed by Mann Whitney U test (unpaired, non-parametric t-test).

839

840

841

842

843 


\section{Figures}

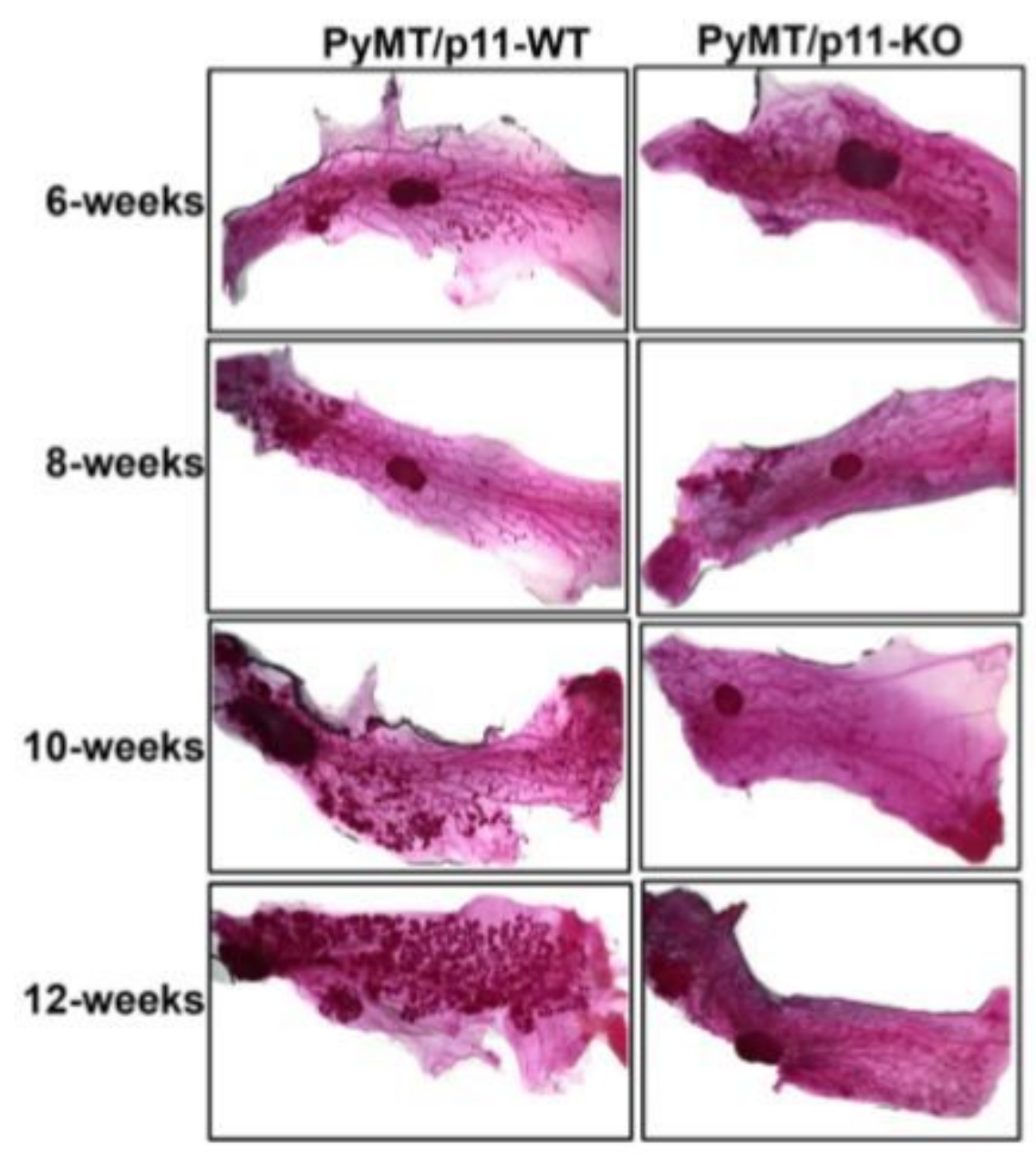

Figure 1

Loss of p11 affects formation of hyperplastic lesions in PyMT driven tumors. Whole mammary glands from PyMT/p11-WT $(n=3)$ and PyMT/p11-KO mice at $(8,10,12$ weeks) were excised, fixed, and stained with Carmine Alum as per standard protocols (See Materials and Methods). Representative images from the 4th abdominal mammary gland from each group and time point is shown. 

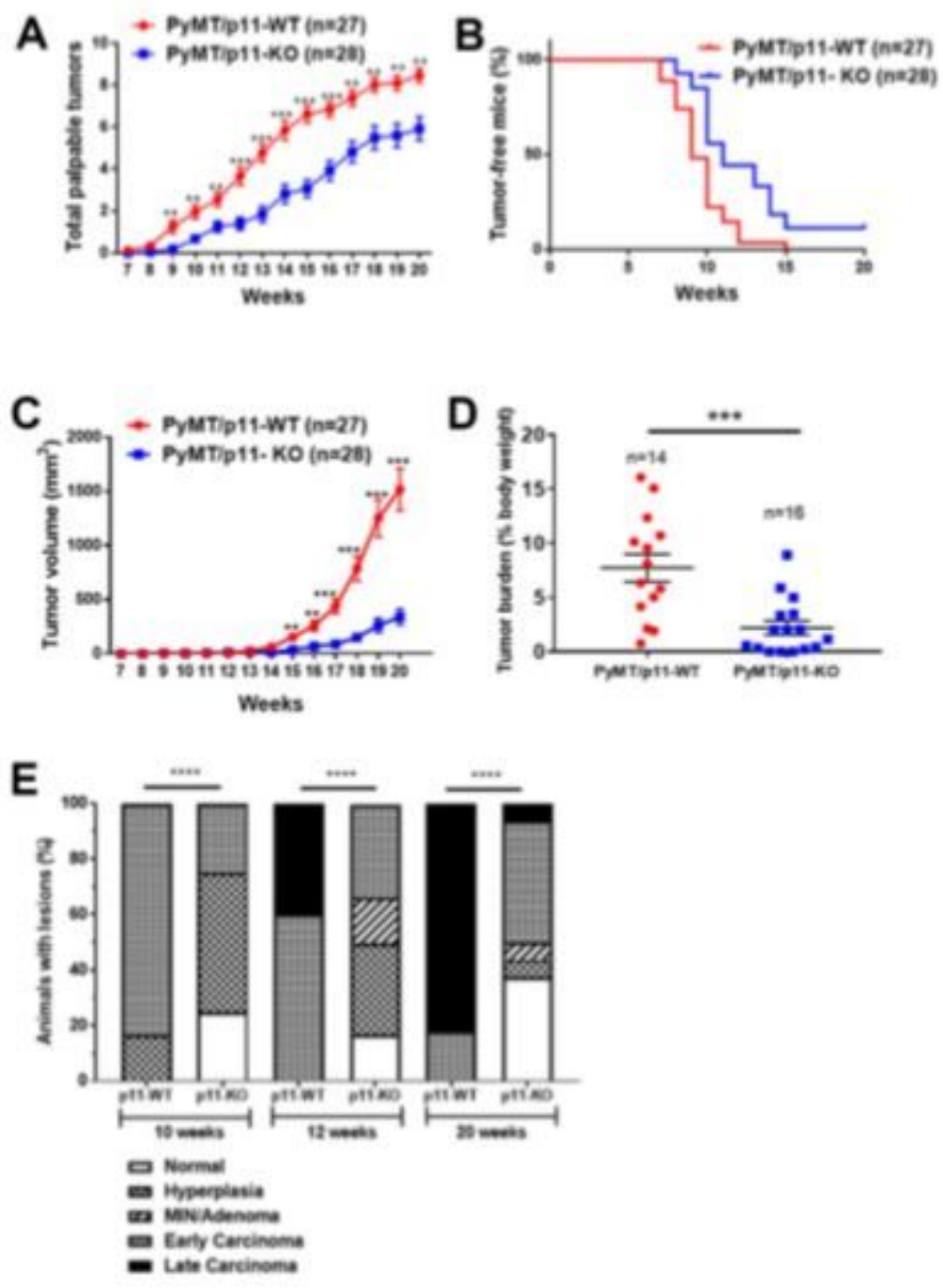

\section{Figure 2}

Tumor initiation, growth, burden, and progression are decreased and delayed in PyMT/p11-KO mice. (A) Appearance of palpable tumors was monitored weekly. (B) Percent tumor-free mice was calculated using Kaplan-Meir analysis (Hazard ration - 2.24, P value -0.0004). (C) Tumor volume was measured weekly using calipers. Total volume was plotted to represent tumor growth rate. (D) Total tumor weight at endpoint (20 weeks) was determined as a percentage of body weight. Data is a compilation of three independent experiments, except (D) which is data from one independent experiment. Significance was determined by non-parametric t-test (Mann Whitney $U$ test - unpaired, non-parametric). (E) Mammary glands and tumors from PyMT/p11-WT and PyMT/p11-KO mice were harvested at 20 weeks, formalinfixed, embedded, and sectioned at $5 \mu \mathrm{m}$ thickness. The H\&E and smooth muscle a-actin-stained sections were classified into histopathological stages by a pathologist in a blinded manner. ( $n=6$ to 12 mice in each group). Statistical analysis was performed by $\chi 2$ - test $(P$ value $<0.0001)$. 


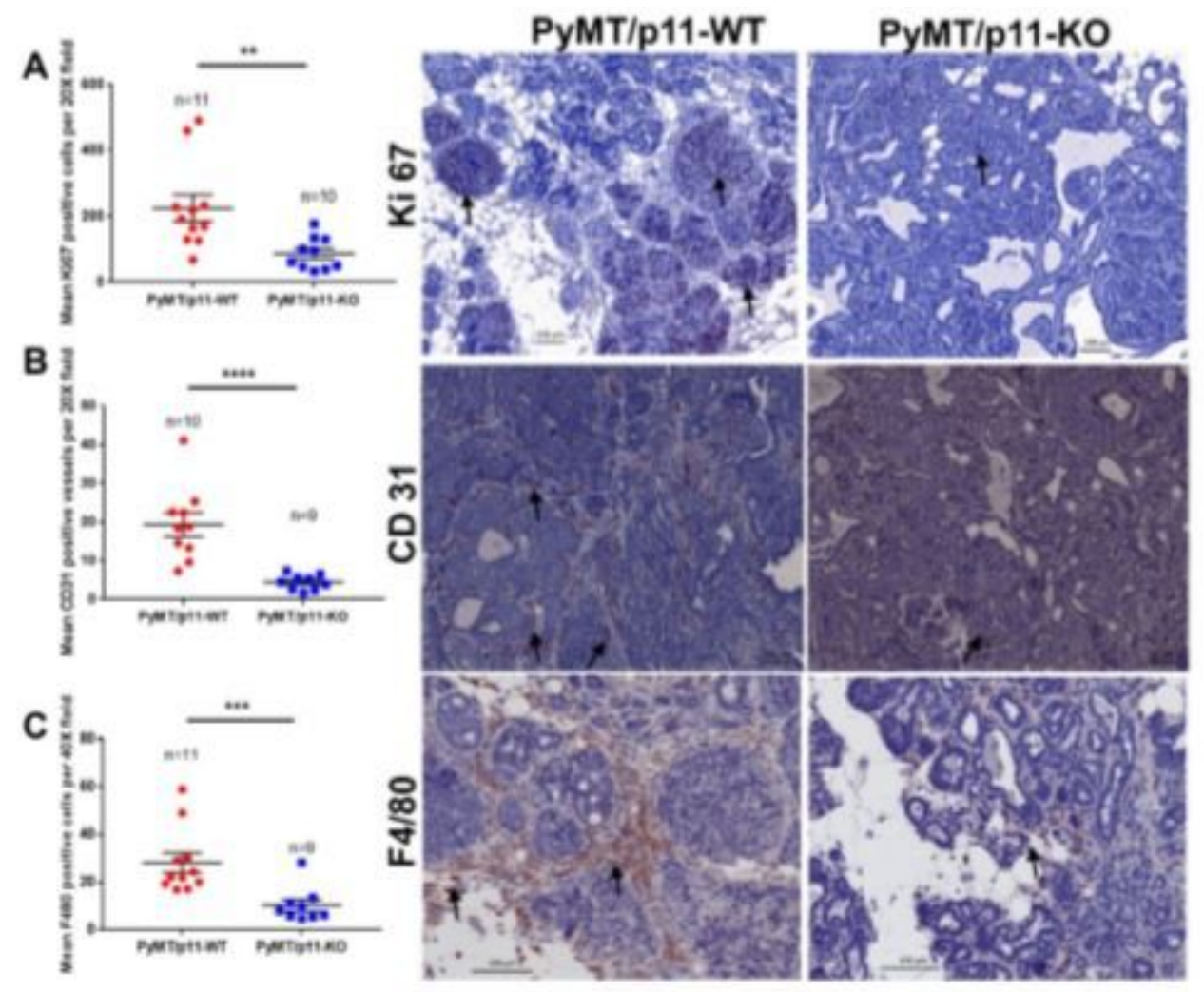

Figure 3

PyMT/p11-KO mice tumors show reduced proliferation vascular density and macrophage infiltration. Formalin-fixed, paraffin-embedded and sectioned tissues from PyMT/p11-WT $(n=11)$ and PyMT/p11-KO $(n=10)$ (end-point, 20 weeks) mice were immunostained for (A) Ki67 (proliferation marker), (B) CD31 (endothelial marker) and (C) F4/80 (mouse macrophage marker). (A) Immunstaining using anti-rabbit Ki67 antibody (Abcam). Stained sections were imaged using Zeiss Zeiss Axio Imager Z1 W/color and monochrome camera at 10X magnification. Left panel: The number of Ki67-positive cells was manually counted using Zen (2012) software in 7-10 random fields per tissue section/mouse. Significance was determined using Mann Whitney $\mathrm{U}$ test (unpaired, non-parametric), $\mathrm{P}$ value is 0.0021 . Right panel: The representative image was captured at 20X magnification. Scale bar - $100 \mu \mathrm{m}$. (B) Immunostaining using anti-rabbit CD31 antibody (Abcam). Stained sections were imaged using Aperio Scanning system (Leica Biosystems, Concord, Ontario) at 40X magnification. Left panel: The number of CD31-positive cells were manually counted using Imagescope software in 7-10 random fields per tissue section/mouse. Mann Whitney $U$ test (unpaired, non-parametric) shows $P$ value $<0.0001$. Right panel: Representative image at 20X magnification. Scale bar - $100 \mu \mathrm{m}$. (C) Immunostaining using anti-rat F4/80 antibody (BM8, Thermofisher Scientific). Stained sections were imaged as in (A), but at 40X magnification. Left panel: The number of F4/80-positive cells were manually counted as in (A). Mann Whitney $U$ test (unpaired, nonparametric) shows $P$ value $<0.0001$. Right panel: Representative image at 20X magnification. Scale bar $100 \mu \mathrm{m}$. 

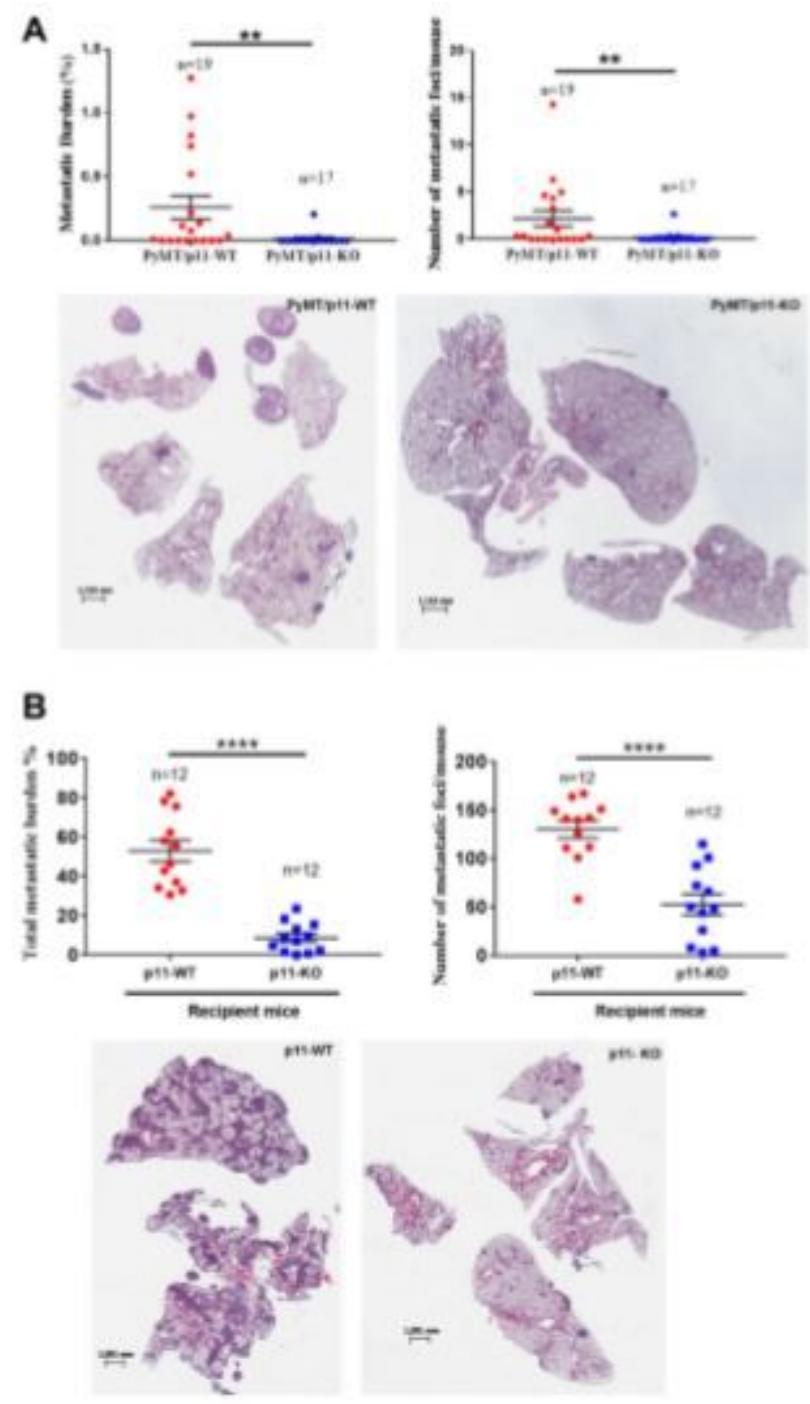

\section{Figure 4}

Metastasis is diminished in PyMT/p11-KO mice. (A) We evaluated pulmonary metastasis at 20-week end point in (spontaneous model) PyMT/p11-WT and PyMT/p11-KO mice by microscopic examination of formalin-fixed, H\&E-stained lung sections $(5 \mu \mathrm{m})$. Three lung sections with each $100 \mu \mathrm{m}$ apart were used for staining. Quantification was performed by Aperio image analysis software (Imagescope). Mean values for three sections were used to calculate the metastatic burden and foci values. (A) Metastatic burden - calculated the total metastatic area and normalized to total lung area (A- upper left panel). Mann Whitney $U$ test show statistical significance with $P$ value of 0.0062 . The number of metastatic foci per mouse lung section was determined by manual counting of imaged sections (A- upper right panel). Mann Whitney $U$ test show $P$ value of 0.0069 . Lower panels: representative lung images from WT and KO mice. (B) Experimental metastasis assay. We injected 2.5x105 Py8119 (p11-WT levels) cells into p11-WT and $\mathrm{p} 11-\mathrm{KO}$ mice ( $\mathrm{n}=12$ mice per group). The lungs were harvested after 14 days, formalin-fixed, and sectioned at $5 \mu \mathrm{m}$ as described above. Mann Whitney non-parametric $\mathrm{t}$ - test for statistical significance was performed. Metastasis was pooled and combined from two independent experiments $(n=6$ each). $P$ value $<0.0001$. Lower panels: representative lung images from WT and KO mice. 


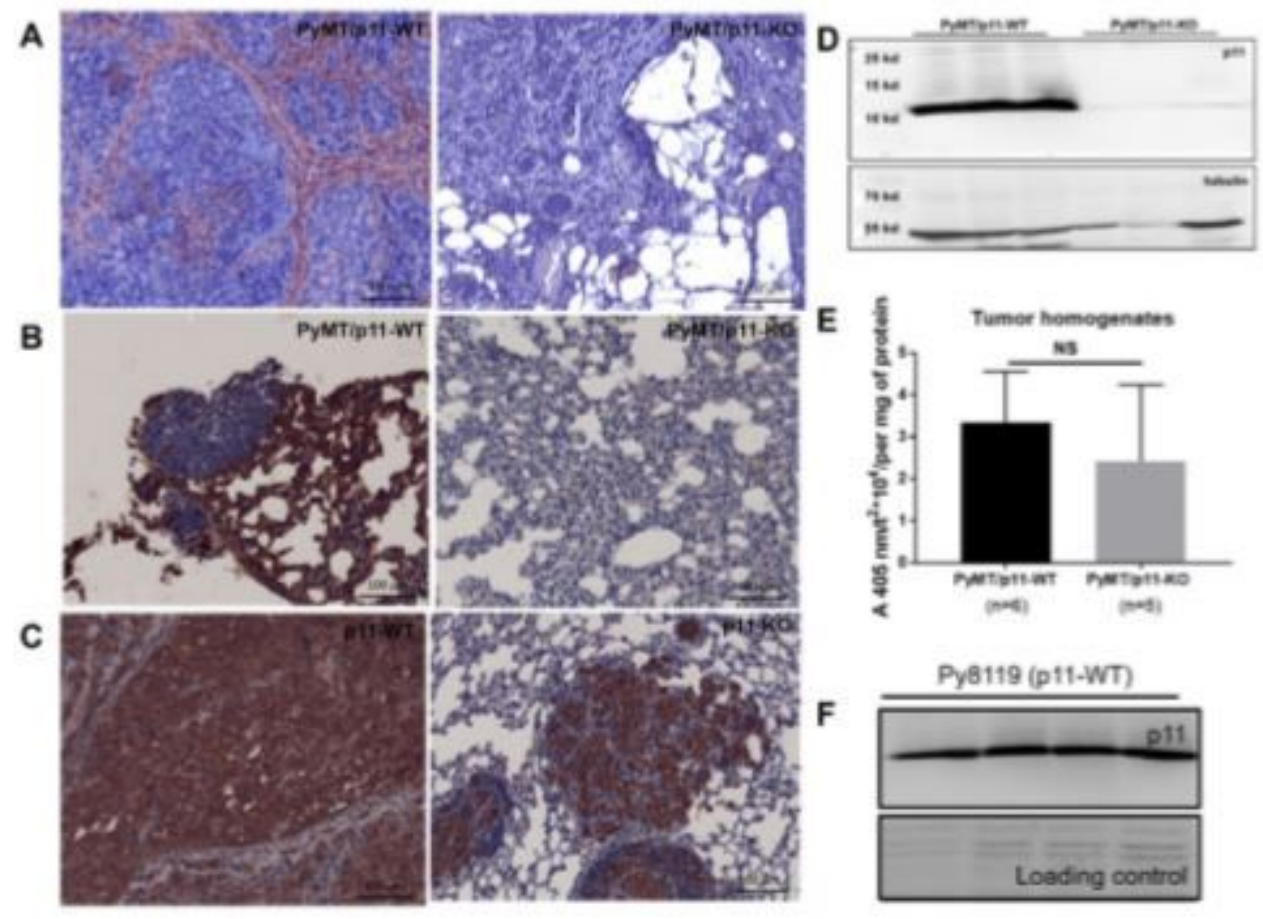

Figure 5

Expression of p11 in mammary and pulmonary metastatic tumors is restricted to the stromal compartment in the PyMT/p11-WT tumors. Immunohistochemical staining was performed using antirabbit p11 antibody (Proteintech), on $5 \mu \mathrm{m}$ sections from 20-week end-point PyMT/p11-WT $(n=10)$ and PyMT/p11-KO tumors ( $n=3)$, and lungs from spontaneous and experimental metastasis assay. (A) Representative images of $\mathrm{p} 11$ immunostained sections of PyMT mammary tumors. As anticipated the tumors from PyMT/p11-KO mice showed no staining, validating the specificity of the antibody. (B) PyMT spontaneous lung metastasis from PyMT/p11-WT and PyMT/p11-KO mice, and (C) experimental metastasis of Py8119 cells injected in p11-WT $(n=4)$ and p11-KO $(n=4)$ mice are shown. Scale bar is 100 $\mu \mathrm{m}$. (D) Western blot of p11 expression in total cell tumor homogenates from PyMT/p11-WT $(n=3)$ and PyMT/p11-KO mice $(n=3)$. (E) Fresh and frozen tumors from both PyMT/p11-WT ( $n=6$ mice) and PyMT/p11-KO ( $n=5$ mice) were homogenized and equal protein $(30-60 \mu \mathrm{g})$ were used for plasmin generation assay as described in Materials and Methods and supplemental section. Mann Whitney $U$ (unpaired, non-parametric) t- test show $P$ value of 0.3290). NS, not significant (F) Western blot for p11 expression in total cell lysates in Py8119 cells obtained from ATCC $(n=4)$ using goat anti-mouse antibody ( $R$ and $D$ systems). 
A
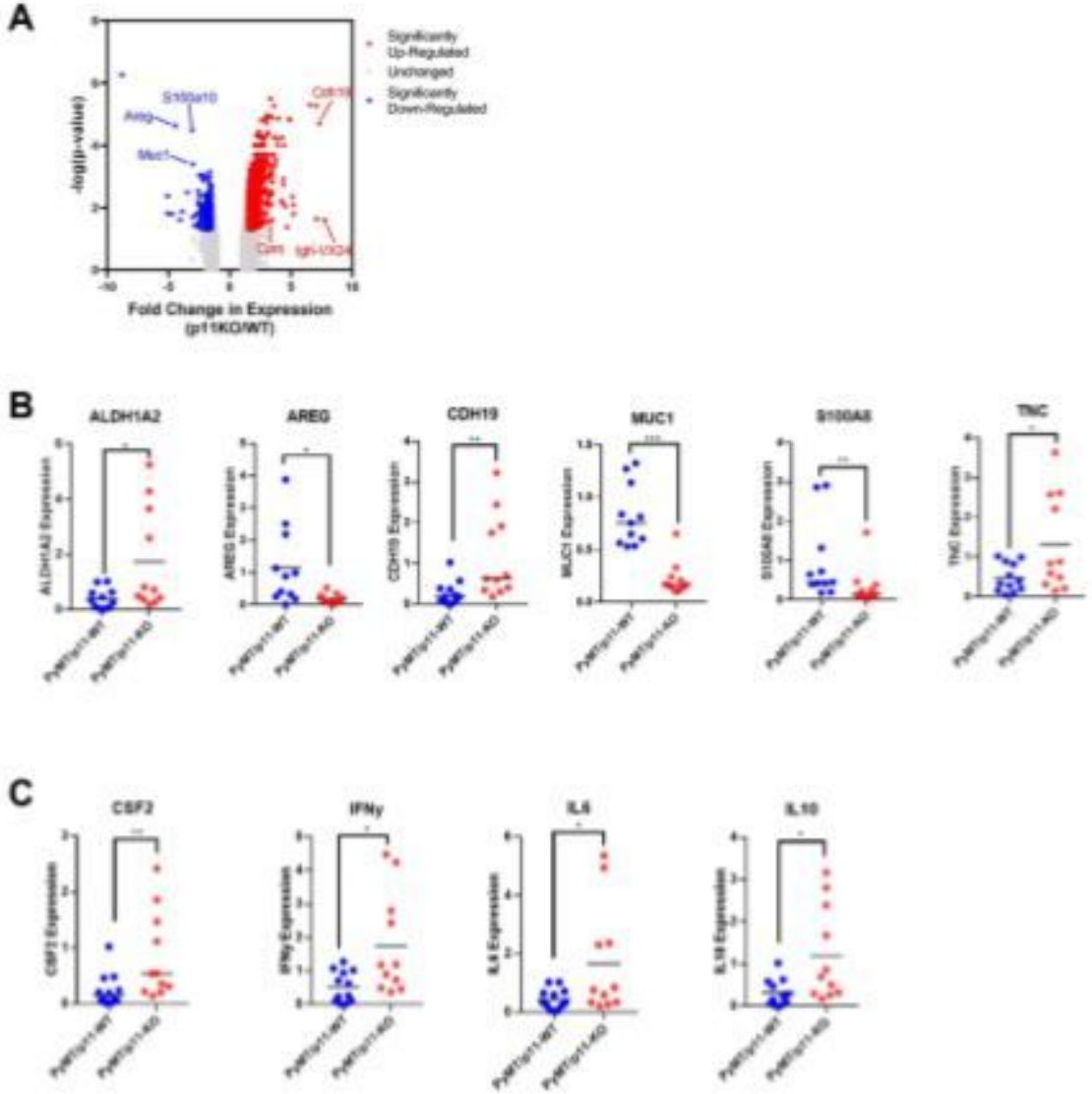

Figure 6

Tumor transcriptome of PyMT/p11-KO mice suggest the downregulation of tumor promoting genes. Gene expression profiling of PyMT/p11-WT and PyMT/p11-KO tumors were performed ( $\mathrm{n}=3$ mice per group). (A) Volcano plot of significantly $P$ value $<0.05)$ upregulated and downregulated genes in the PyMT/p11 $\mathrm{KO}$ mice ( $\mathrm{n}=3$ mice in each group). The top 6 genes are highlighted. Genes which were up- or downregulated more than 2 -fold $(\log 2=0.678)$ at a significance level of $P$ value $<0.05$ were considered differentially expressed. (B) Validation of the top 9 differentially expressed genes using quantitative RTPCR (10-11 mice per group). Significance was determined by unpaired t- test. Only the genes significantly altered are shown. (C) Loss of p11 in PyMT tumors results in differential cytokine expression profile. We performed quantitative RT-PCR on tumors isolated from PyMT/p11-WT and PyMT/p11 KO mice (10-11 mice per group), using a CFX96 or CFX384 Touch Real-Time PCR Detection system (BioRad). Relative mRNA expression was log-2 transformed prior to plotting and statistical analysis. Significance was determined by unpaired, $\mathrm{t}$ - test. Only those cytokines significantly altered are shown. 

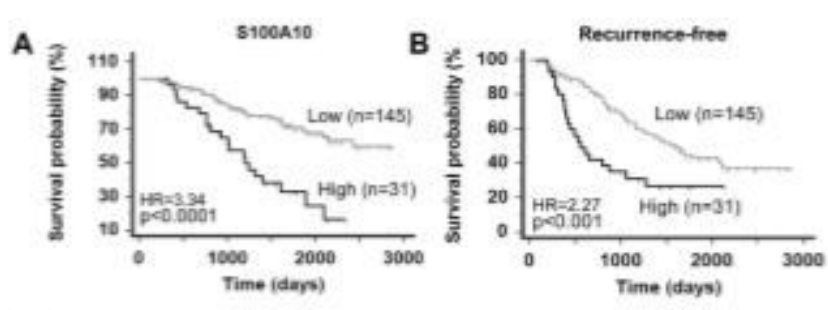

c
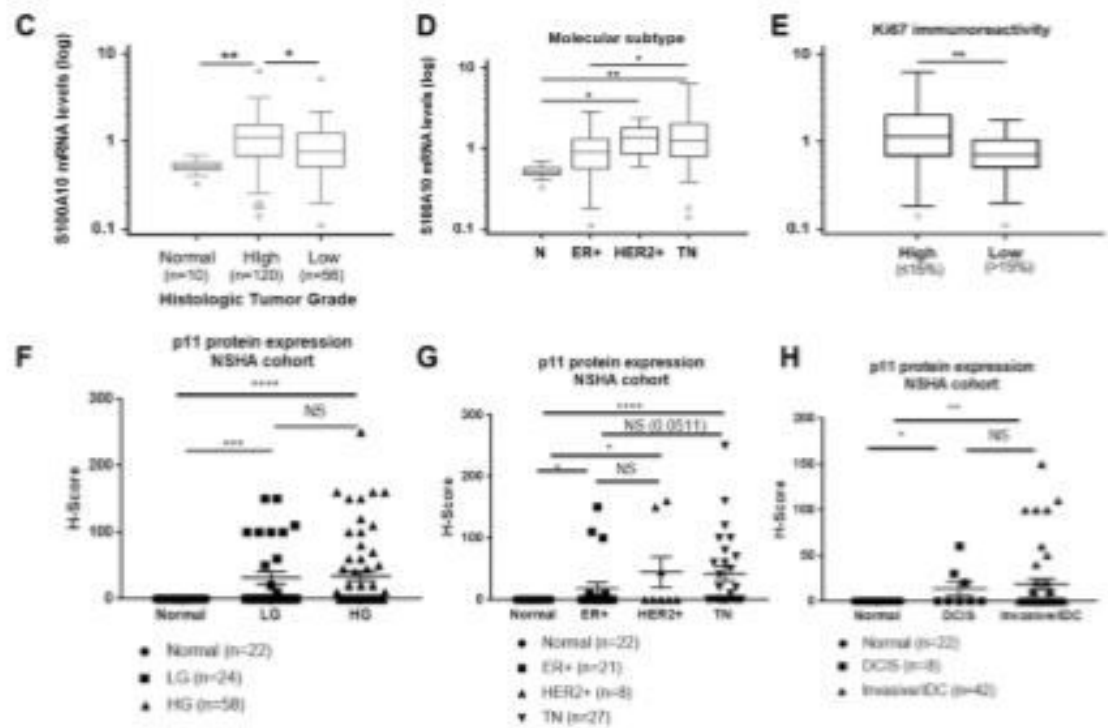

Figure 7

Expression of S100A10 (p11) expression in breast cancer patients. Treatment-naïve primary breast cancer samples $(n=176)$ were obtained through the CBCF Tumor Bank and used for gene expression analysis. S100A10 high and low mRNA levels were based on the signal intensity from our gene expression microarray profile using the Receiver Operating Characteristic (ROC) curve analysis. Correlation of S100A10 (p11) mRNA with (A) overall survival probability. High mRNA levels of p11 gene (p11) are significantly associated with poor patient overall survival (hazard ratio of 3.34). (B) Recurrence free survival, with hazard ratio 2.27. (C) For histological tumor grade, p11 is significantly upregulated in high grade tumors compared to normal breast and low-grade tumor tissues. (D) For molecular subtype, p11 is significantly up-regulated in HER2+ and triple negative breast cancers compared to normal breast tissues. (E) In breast cancer patients, p11 mRNA levels are significantly higher in tumors with high Ki67 immunoreactivity. S100A10 (p11) protein is overexpressed in breast tumors compared to normal mammary tissues. $(F, G, H)$ Immunohistochemical staining of p11 in normal breast tissues and in low grade (LG), high grade (HG), ductal carcinoma in situ (DCIS), IDC/Invasive, ER+, Her2+ and Triple negative (TN) tumors was performed using anti-p11 antibody (Proteintech). These human tissues were obtained from Queen Elizabeth II Health Sciences Centre, Nova Scotia Health Authority (see Materials and Methods). The stained sections were scored (semi-quantitative) based on percent positive tumor cells and intensity of staining by a pathologist (blinded). $\mathrm{H}$ score was determined based on the formula described and the samples/tissues were separated and plotted based on (F) clinical/histological grade, (G) pathological grade, and $(\mathrm{H})$ molecular subtype. Statistical analysis was performed by Mann Whitney $\mathrm{U}$ test (unpaired, non-parametric t-test). 